\title{
Combined Support-Vector-Machine-Based Virtual Screening and Docking Method for the Discovery of IMP-1 Metallo- $\beta$-Lactamase Inhibitors
}

\author{
Jiao Chen, Yifang Liu, Mi Fang, Hui Chen, Xingzhen Lao, Xiangdong Gao, Heng Zheng*, Wenbing Yao* \\ School of Life Science and Technology, China Pharmaceutical University, Nanjing, P.R. China \\ Author contributions: Jiao Chen, Yifang Liu and Mi Fang contributed equally to this work \\ *Corresponding author: zhengh18@hotmail.com, wbyao@cpu.edu.cn(W. Yao)
}

Received November 07, 2013; Revised November 13, 2013; Accepted November 15, 2013

\begin{abstract}
Metallo- $\beta$-lactamases can hydrolyze a broad range of $\beta$-lactam antibiotics and no effective inhibitors could be used in the clinic. Therefore, the discovery of metallo- $\beta$-lactamase inhibitors has attracted much attention in recent years. In this study, a support vector machine (SVM) that separates compounds into positives and negatives, combined with docking method was employed for virtual screening of IMP-1 metallo- $\beta$-lactamase inhibitors. Eight of the twenty five selected compounds were purchased for in vitro assays. Among them, four compounds show inhibitory potency against IMP-1. Two of them are found to have novel scaffolds, implying a good potential for further optimization.
\end{abstract}

Keywords: IMP-1 metallo- $\beta$-lactamase inhibitors, support vector machine, docking, virtual screening, in vitro assays

Cite This Article: Jiao Chen, Yifang Liu, Mi Fang, Hui Chen, Xingzhen Lao, Xiangdong Gao, Heng Zheng, and Wenbing Yao, "Combined Support-Vector-Machine-Based Virtual Screening and Docking Method for the Discovery of IMP-1 Metallo- $\beta$-Lactamase Inhibitors.” American Journal of Biomedical Research 1, no. 4 (2013): 120-131. doi: 10.12691/ajbr-1-4-8.

\section{Introduction}

The production of $\beta$-lactamases is one of the most important resistant mechanisms in bacteria, which can inactivate $\beta$-lactams by hydrolyzing the amide bond of the four-membered $\beta$-lactam ring. These enzymes can be divided into serine- $\beta$-lactamases and metallo- $\beta$-lactamases (MßLs), which employ a serine and one or two zinc ions in the active site, respectively [1]. Except monobactams, the MBLs can degrade almost all classes of $\beta$-lactams including carbapenems, which are stable against the serine- $\beta$-lactamases. The currently clinical used $\beta$ lactamase inhibitors such as clavulanic acid, sulbactam, and tazobactam can poorly inhibit MBLs.

Imipenemase (IMP), one of the most dangerous metallo$\beta$-lactamases, can hydrolyze a broad spectrum of lactams, including penicillins, cephalosporins, and carbapenems [2]. Accordingly, 47 variants of IMP M $\beta$ Ls have been identified (http://www.lahey.org/Studies/other.asp\#ttable1, data collected before 29 Oct. 2013), and the number is rapidly growing. In the past few years, several types of compounds have been reported inhibitory activity against IMP-1, such as 2, 3-disubstituted succinic acids [3], thiols [4], thioesters [5], maleic acid derivatives [6], and carbapenem derivatives [7] etc. However, none of these inhibitors has fortunately passed clinical trials. Thus, the discovery of new types of $\mathrm{M} \beta \mathrm{L}$ inhibitors has increasingly attracted public attention in recent years in order to recover the effectiveness of $\beta$-lactam antibiotics.

Several in silico methods including pharmacophore [8], docking [9], molecular dynamics [10] and our previously established 3D-QSAR models [11] have been used for facilitating the search and design of IMP M $\beta \mathrm{L}$ inhibitors. These methods have shown significant capability in the identification of potential IMP inhibitors. Molecular docking, which is the most widely used structure-based virtual screening method, considers sufficiently the match of three-dimensional geometrical shape between the ligand and the active site of receptor [12], but the problems such as the sparse chemical space, the highly consuming of time and computing resource, and even the limited diversity of training set may affect their applications. Thereby, it is highly desirable to obtain other computational model capable of screening large compound libraries rapidly and guiding the discovery of IMP inhibitors with new scaffolds.

Support Vector Machine (SVM), a supervised learning method used for classification, has been gaining popularity due to many attractive features and promising empirical performance in the fields of protein structure prediction [13,14], specific biochemical reaction sites identification [15,16,17], protein-ligand binding affinity prediction [18] and so on. Prof. Chen. Y. Z. et.al employed this method in virtual screening of kinase inhibitors [19], gamma-secretase inhibitors [20], HDAC inhibitors [21], new potential organocatalysts [22], Abl 
inhibitors [23], and small molecule aggregators [24]. They demonstrated that SVM-based virtual screening has comparable and in some cases substantially higher yields than those of conventional virtual screening tools reported in the literature. Most importantly, it is in a timely, lowresource manner. SVM trains models and identifies active compounds based on the physicochemical properties rather than their structural similarity. So no knowledge of enzyme structure, no computation of structural flexibility, binding affinities, activity-related features and no inhibitory mechanisms are required. However, SVMbased virtual screening still perform imperfectly in some aspects, for example, in reducing the false positive rate, which might originates from the lack of consideration of the information about the macromolecule target. Therefore, it is expected the combined SVM-based virtual screening and docking method can lead to a high hit rate and a large enrichment factor, as well as high-speed [25].

In this work, we adopted the combined SVM-based virtual screening and docking method for new active inhibitor discovery. The accuracy of SVM is largely dependent on the descriptors used in the model. So we applied the automatic step-by-step descriptors selector (SDS-SVM) developed in house to select the molecular descriptors. The SVM model was built based on the known IMP-1 inhibitors and putative noninhibitors, which could rapidly classify the compounds in the library into potential IMP-1 inhibitors and noninhibitors. The hit compounds were subjected to subsequent docking screening. Finally, we selected 25 compounds and 8 were purchased for in vitro assays. The schematic workflow is shown in Figure 1.

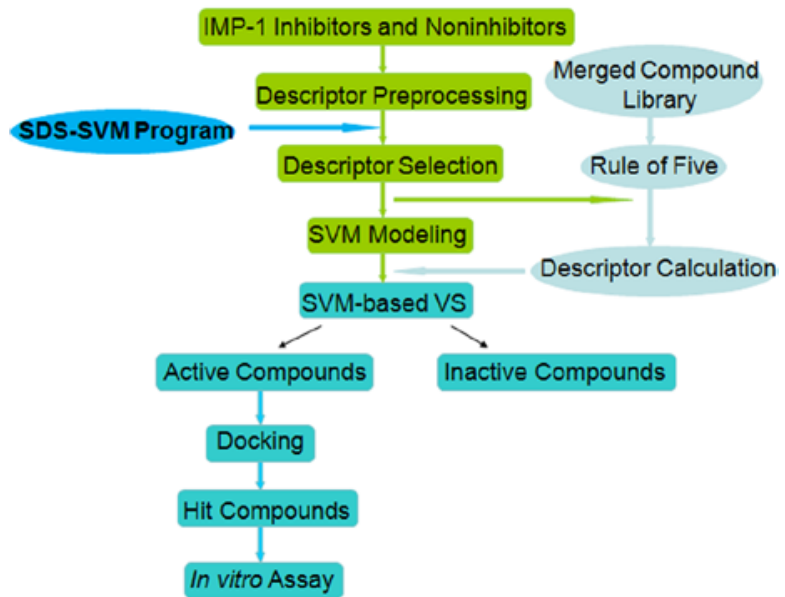

Figure 1. A schematic workflow illustrating the process of SVM modeling and VS.

\section{Experimental Procedures}

\subsection{Data Sets}

A total of 262 IMP-1 inhibitors were collected from different literature (Supplementary Data, Table S1) [3,4,5,6,7,26-38]. Scaffolds of the representative inhibitors are shown in Figure 2. As few IMP-1 noninhibitors have been reported, putative noninhibitors [39] were generated according to the method described in our previous study [40]. 10,605 putative noninhibitors were randomly selected from putative negative families for SVM modeling.

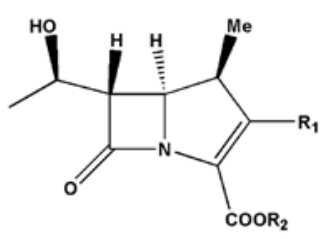

Carbapenem Derivatives<smiles>[R]C(=O)S[C@H]([R2])C(=O)O</smiles>

Thioester Derivatives<smiles></smiles><smiles>[R]c1ccccc1CSOCCOCCOCCO</smiles>

Thiol Compounds
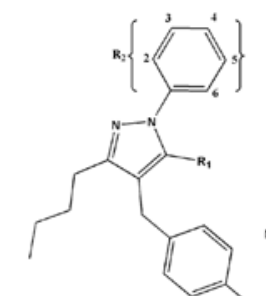

Biphenyl Tetrazoles<smiles>C=CCC1[C@H](C)[C@@H](C(=O)O)[C@@H](C)C1(Cl)Cl</smiles>
Cyclobutanone Analogues<smiles>[R]C(=O)NN=C</smiles>

$\mathrm{N}$-Acylhydrazones

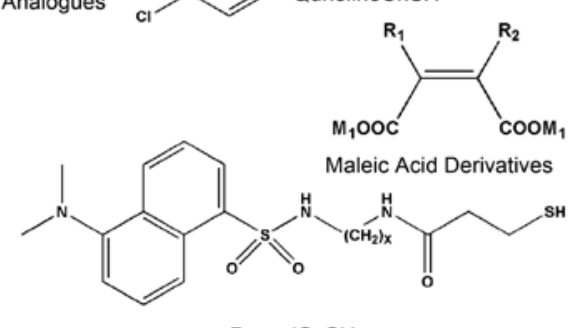

DansylCnSH

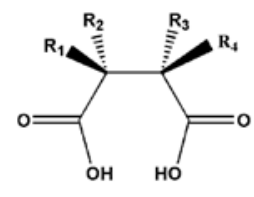

Succinic Acids<smiles>[R]c1ccc(C(=O)O)c(C(=O)O)c1P</smiles>

Phthalic Acid Derivatives

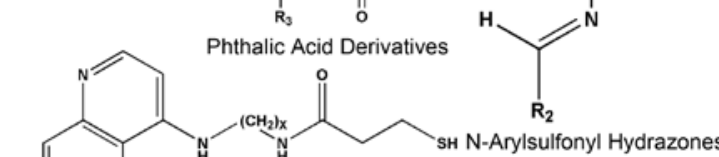

SB238569

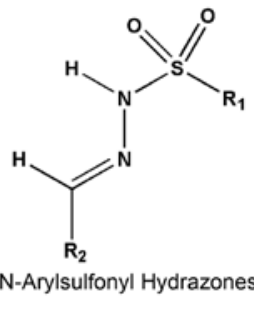

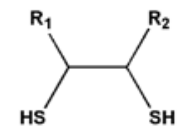

Thiol Compounds

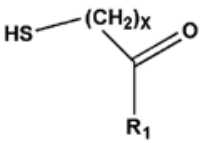

Thiol Compounds

Figure 2. Scaffolds of the representative IMP-1 inhibitors

\subsection{Descriptor Selection}

Descriptor selection has an important impact on the improvement of classification performance in SVM modeling. In this work, we applied the automatic step-by- step descriptor selector (SDS-SVM) developed in house [40] based on Perl \& R to select the molecular descriptors for SVM modeling.

The molecular descriptors used in our work were calculated in MOE2009 software. Redundant descriptors with $>90 \%$ identical values and those irrelevant to the 
pharmacokinetic and toxicological properties were removed in the preprocessing. A stricter criterion was used to define the positive compound in order to select more representative features of the inhibitors sensitively. Thus, only 123 compounds with $\mathrm{IC}_{50} \leq 10 \mu \mathrm{M}$ or $\mathrm{Ki} \leq 2$ $\mu \mathrm{M}$ among the total 262 IMP-1 inhibitors were considered as positives, whereas the other 139 compounds with $\mathrm{IC}_{50}>$ $10 \mu \mathrm{M}$ or $\mathrm{Ki}>2 \mu \mathrm{M}$ were treated as negatives during the descriptor selection step. After step-by-step eliminating, 24 descriptors were retained for the subsequent virtual screening. These descriptors characterize structural and physicochemical features of IMP-1 inhibitors.

\subsection{SVM Modeling and Virtual Screening}

In our SVM model, 225 IMP-1 inhibitors with IC $_{50} \leq$ $200 \mu \mathrm{M}$ or $\mathrm{Ki} \leq 100 \mu \mathrm{M}$ together with 10605 putative noninhibitors were divided into the training set (180 positives and 8484 putative noninhibitors) and test set (45 positives and 2121 putative noninhibitors) based on their structural diversity and biological activity. The model was built based on the training compounds and then tested using the independent test set. Subsequently, the merged compound library was subjected to virtual screening based on the selected 24 descriptors by this SVM model. 2861 compounds were hit.

\subsection{Molecular Docking}

The SVM-hit compounds were washed and the lowest energy conformer of each compound was generated in MOE2009 (Chemical Computing Group Inc., Montreal, Canada). Molecular docking studies were carried out by GOLD (Genetic Optimization of Ligand Docking) 3.0.1 [41]. GOLD adopts the genetic algorithm to dock flexible ligands into protein binding sites. The crystal structure of IMP-1 in complex with 2,3-bis-benzo[1,3]dioxol-5ylmethyl- succinic acid (PDB entry: 1JJE) was used as the reference receptor for the docking studies. The crystal waters were deleted and hydrogen atoms were added to the protein. Residues that stay within $15 \AA$ from the original ligand were defined as the binding site. A tetrahedral geometry was applied to $\mathrm{Zn} 1$ (3H binding site), whereas a trigonal bipyramid was set to $\mathrm{Zn} 2(\mathrm{DCH}$ binding sites). The default values were used for the remaining parameters, and 10 Genetic Algorithm runs were performed for each ligand. GOLDScore was used to evaluate the docking results.

\subsection{In vitro IMP-1 Inhibitory Assay}

The IMP-1 gene lacking the first 18 residues was synthesized chemically and then cloned between BamHI and XhoI restriction sites into the pET-28a plasmid harboring a kanamycin resistance gene. The forward primer was 5'CCGggatccGCAGAGTCTTTGCCAGATTTAAAAA-3'

and the reverse primer was 5'CCCGctcgagTTAGTTGCTTGGTTTAGATGGTTTTT-3’. The constructed plasmids encoding IMP-1 was transferred into E.coli BL21 (DE3). The enzymes were expressed and purified as previously described [42].

Inhibition of IMP-1 was monitored by following the absorbance variation that resulted from the hydrolysis of the $\beta$-lactam ring. All the kinetic assays were performed at $30^{\circ} \mathrm{C}$ in the buffer of $50 \mathrm{mM}$ Hepes, $100 \mu \mathrm{M} \mathrm{ZnCl} \mathrm{Zn}_{2}$ and $0.1 \mathrm{M} \mathrm{NaCl}$ at $\mathrm{pH}$ 7.5. The hydrolysis of ampicillin was measured as the substrate at 235nm, using a UV-1800 spectrophotometer (MAPADA). The enzyme and inhibitor were preincubated at $30^{\circ} \mathrm{C}$ for $20 \mathrm{~min}$ before the substrate was added. The concentration of the tested compound which exhibited $50 \%$ inhibition of IMP-1 was set as IC $_{50}$.

\section{Results and Discussion}

\subsection{Descriptor Selection}

All the collected 262 IMP-1 inhibitors retrieved from the literature were used for descriptor selection by our SDS-SVM program. The inhibitors were divided into 123 positives and 139 negatives (Supplementary Data, Table S1). After SDS-SVM elimination, 24 descriptors survived for SVM modeling and virtual screening. The distribution of them can be seen in Table 1. These descriptors cover eight classes including physical properties, subdivided surface areas, atom counts and bond counts, adjacency and distance matrix descriptors, pharmacophore feature descriptors, partial charge descriptors, potential energy descriptors and surface area, volume and shape descriptors. Among the 24 selected descriptors, 9 belong to the class of surface area, volume and shape descriptors, which depend on the structure connectivity and conformation (dimensions are measured in $\AA$ ), indicating that this class contributes the most to the SVM model we will build.

Table 1. Molecular Descriptors Selected by the SDS Algorithm for SVM-based VS

\begin{tabular}{lcl}
\hline Descriptor class & $\begin{array}{c}\text { Number of descriptors in } \\
\text { each class }\end{array}$ & Descriptors \\
\hline Physical Properties & 2 & FCharge, rsynth \\
Subdivided Surface Areas & 2 & SlogP_VSA8, SlogP_VSA9, SMR_VSA7 \\
Atom Counts and Bond Counts & 2 & a_ICM, opr_leadlike \\
Adjacency and Distance Matrix Descriptors & 2 & BCUT_PEOE_0, petitjean \\
Pharmacophore Feature Descriptors & 3 & vsa_acc, vsa_other \\
Partial Charge Descriptors & 1 & PEOE_VSA+2, PEOE_VSA-4, PEOE_VSA-6 \\
Potential Energy Descriptors & 9 & E_stb \\
Surface Area, Volume and Shape Descriptors & rgyr, vsurf_DW13, vsurf_EWmin3, vsurf_W4, vsurf_HB1, & vsurf_HB4, vsurf_EDmin2, vsurf_ID4, vsurf_DD12 \\
\hline
\end{tabular}


SVM training algorithm builds a model that predicts whether a new compound falls into one category or the other [43]. In our SVM model, the training set comprises 8,664 compounds, including 180 known IMP-1 inhibitors (positives) and 8,484 putative noninhibitors (negatives). The 24 selected descriptors were used to build the SVM model (Table 2). The accuracies for predicting IMP-1 inhibitors (SE) and noninhibitors (SP) are $89.44 \%$ and 99.98\%, respectively. The overall prediction accuracy Q and Matthews correlation coefficient (MCC) are 99.76\% and 0.94, respectively.

Fivefold cross-validation was performed to evaluate the generated SVM model. As shown in Table 2, the average accuracies for predicting IMP-1 inhibitors (SE) and noninhibitors (SP) are $81.67 \%$ and $99.92 \%$, respectively. The average overall prediction accuracy Q and MCC are $99.54 \%$ and 0.88 , respectively. These indicate that the generated SVM model is reliable for the prediction of training set agents.

Subsequently, the established SVM classification model was further validated by an independent test set, with the purpose of assessing the predictive power of SVM model to the external compounds that are not contained in the training set. The independent test set contains 45 inhibitors and 2,121 noninhibitors. Of the 45 inhibitors, 40 were correctly predicted, indicating a prediction accuracy of $88.89 \%$ (SE, Table 2) for the inhibitors. For the 2,121 noninhibitors, all of them were properly predicted, indicating a prediction accuracy of $100 \%$ (SP, Table 2). The overall prediction accuracy Q and MCC are 99.77\% and 0.94 , respectively, which is comparable with the training set. All of these results demonstrate that our SVM model is reliable in differentiating the IMP-1 inhibitors and noninhibitors in both the training set and the external test set.

Table 2. Validation results for the SVM model by a fivefold cross-validation method and an independent test set method

\begin{tabular}{|c|c|c|c|c|c|c|c|c|c|}
\hline Method & group & $\mathrm{TP}$ & FN & $\mathrm{SE}(\%)$ & $\mathrm{TN}$ & $\mathrm{FP}$ & $\mathrm{SP}(\%)$ & $\mathrm{Q}(\%)$ & MCC \\
\hline SVM model & & 161 & 19 & 89.44 & 8483 & 2 & 99.98 & 99.76 & 0.94 \\
\hline \multirow{6}{*}{ fivefold cross-validation } & 1 & 28 & 8 & 77.78 & 1696 & 0 & 100.00 & 99.54 & 0.88 \\
\hline & 2 & 31 & 5 & 86.11 & 1694 & 2 & 99.88 & 99.60 & 0.90 \\
\hline & 3 & 29 & 7 & 80.56 & 1692 & 4 & 99.76 & 99.36 & 0.84 \\
\hline & 4 & 26 & 10 & 72.22 & 1696 & 0 & 100.00 & 99.42 & 0.85 \\
\hline & 5 & 33 & 3 & 91.67 & 1695 & 1 & 99.94 & 99.77 & 0.94 \\
\hline & Average & & & 81.67 & & & 99.92 & 99.54 & 0.88 \\
\hline Independent validation test & & 40 & 5 & 88.89 & 2121 & 0 & 100.00 & 99.77 & 0.94 \\
\hline
\end{tabular}

Virtual screening of the merged library with 1.257 million compounds by the established SVM model generated 6,452 hits, which were later subjected to a more accurate docking program.

\subsection{Molecular Docking}

Docking is the most popular structure-based virtual screening method. Among various docking programs, GOLD has been proved to be a better program in the docking of IMP-1 inhibitors, giving a good correlation between the experimentally determined affinities and the GOLD scores [9]. Therefore, we chose GOLD to re-screen the SVM-hit 2,861 compounds. Twenty five compounds were selected based on experience and the GOLD scores for further investigation. Eight of them were purchased for in vitro assays, since the others were not commercially available. The docking scores and calculated MM/GBVI binding free energy values are shown in Table 3.

Table 3. Docking scores, MM/GBVI and $\mathrm{IC}_{50}$ values of the assayed compounds

\begin{tabular}{cccc}
\hline Compound & GoldScore & MM/GBVI $(\mathrm{kcal} / \mathrm{mol})$ & IC $_{50}(\mu \mathrm{M})$ \\
\hline 1 & 71.27 & -124.36 & 124 \\
2 & 73.85 & -25.62 & 227 \\
3 & 75.57 & -124.94 & 254 \\
4 & 85.86 & -119.29 & 288 \\
5 & 80.07 & -113.79 & 7800 \\
6 & 73.65 & -25.86 & $\mathrm{ND}^{\mathrm{a}}$ \\
7 & 73.08 & -127.70 & $\mathrm{ND}$ \\
8 & 99.60 & -31.89 & $\mathrm{ND}$ \\
\hline a Not detected because of poor solubility & &
\end{tabular}

\subsection{In vitro Assay}

IMP-1 was expressed and purified, and then confirmed by SDS-PAGE (Figure 3). Inhibitory activities of the purchased compounds were determined using ampicillin as report substrate by the methods described above. Their structures together with the measured $\mathrm{IC}_{50}$ values are shown in Figure 4.

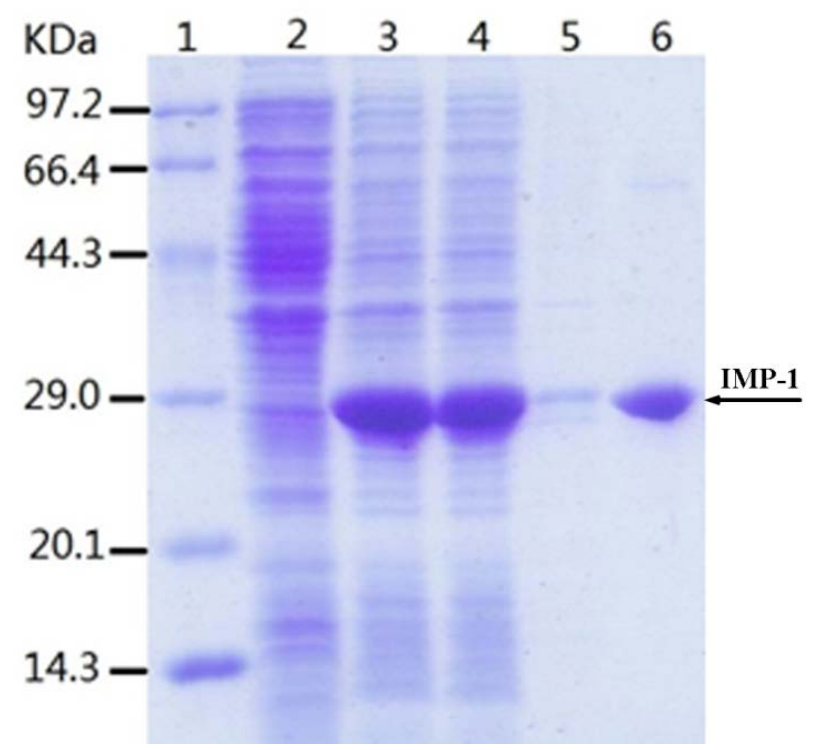

Figure 3. SDS-PAGE gels of IMP-1 purification. Lane 1, Takara protein molecular weight marker; Lane 2, crude proteins before induced by IPTG; Lane 3, crude proteins after inducement and ultrasonication; Lane 4 , crude proteins after ultrasonication and centrifugation in supernatant

${ }^{\mathrm{a}}$ Not detected because of poor solubility 
<smiles></smiles><smiles>O=C(O)CSCSCC(=O)O</smiles>
3 $\mathrm{IC}_{50}=254 \mu \mathrm{M}$<smiles>O=C(CSc1n[nH]c(=O)n1-c1ccc(OCc2ccccc2)cc1)c1ccccc1</smiles><smiles>[14CH3][14CH3]</smiles><smiles>Cc1nc(-c2ccc(S(=O)(=O)NCc3ccco3)s2)cs1</smiles>

2<smiles>[14CH3][14CH3]</smiles><smiles>O=C(O)CC(NC(=O)Cc1cccc2ccccc12)C(=O)O</smiles>

4

$$
\mathrm{IC}_{50}=288 \mu \mathrm{M}
$$<smiles></smiles><smiles>O=S(=O)(CS(=O)(=O)NCCc1ccc(Cl)cc1)NCCc1ccc(Cl)cc1</smiles>

8<smiles>O=C(O)CC(C(=O)O)c1ccccc1</smiles>

\section{5}

$$
\mathrm{IC}_{\mathbf{5 0}}: \mathrm{ND}
$$

Figure 4. Compounds obtained by using the combined VS method together with their measured bioactivities. ND: not detected

The docking scores and calculated binding free energies are not always consistent with the experimental bioactivities. The values of MM/GBVI are comparable only when the evaluated compounds have similar binding manner, for example, the MM/GBVI values of compound 1, 3, 4, 5 and 7 with two carboxyl groups in their structures, are distinct from the values of compound 2, 6 and 8 without carboxyl groups. Table 3 also demonstrates that the top ranked compound $\mathbf{5}$ with GoldScore 80.07 has almost no inhibitory capacity against IMP-1, while for compound 1, whose GoldScore is only 71.27, possesses much higher inhibitory capacity with IC $_{50}$ value of 124 $\mu \mathrm{M}$. The results suggest that it is not always successful to select the inhibitor solely based on the docking score or binding energy. The better way is to combine different methods to improve the accuracy and sensitivity.

\subsection{Analysis of Active Compounds}

An in vitro IMP-1 inhibitory assay was carried out for the purchased 8 compounds. The inhibitory potency was tested with ampicillin as the substrate. Four compounds showed $\mathrm{IC}_{50}<300 \mu \mathrm{M}$ and three compounds were not tested because of their poor solubility (Figure 4). Among them, compound $\mathbf{1}$ and $\mathbf{3}$ have new scaffolds whereas compound $\mathbf{2}$ possesses common pharmacophore of sulfonamide with the reported inhibitor of DansylCnSH, and compound $\mathbf{4}$ is a new derivative of succinic acid.
These four active compounds were redocked into IMP-1 metallo- $\beta$-lactamase (PDB code: $1 \mathrm{JJE}$ ). Their individual binding interactions are shown in Figure 5. The residues were numbered according to Galleni et al. [44]. Docking results reveal that compound $\mathbf{1}, \mathbf{3}$ and $\mathbf{4}$ share similar binding mode with two carboxyl groups coordinating to the two zinc ions of IMP-1. Zn2 coordinates to both of the two carboxyl groups while $\mathrm{Zn} 1$ coordinates to only one of them. The sidechains of Asn233 and Lys224 act as hydrogen bond donor to the carboxyl groups in compound 1, 3 and 4. Arene-arene interactions were generated between imidazole group of His in IMP-1 and aromatic rings in the active compounds. For compound 2, the furan ring also formed arene-arene interaction with the indole group of Trp64. Zn1 of IMP-1 coordinates to the oxygen of sulfonamide and Zn2 coordinates to both of the oxygen and nitrogen of sulfonamide in compound 2 .

For the sake of increasing inhibitory activity of these compounds, chemical modifications are underway. Protein-ligand interaction fingerprint (PLIF) analysis of the crystal structures of IMP-1 in complex with inhibitors [40] suggests that the hydrogen bond between the inhibitor and Asn233 is an important interaction. However, for compound 1, no hydrogen bond is formed with Asn233. Substitutions like - $\mathrm{OH}$ on the benzene ring at $\mathrm{R} 2$ might increase the potential inhibitory activity of compound 1. Take compound 3 as another example, our 3D-QSAR model [40] suggested that modifications with 
some hydrophobic substituent groups might enhance its inhibitory activity against IMP-1.

\section{Conclusion}

M $\beta$ Ls have gained a lot of attention owing to their broad hydrolytic spectrum on $\beta$-lactams and the current lack of effective inhibitors. In addition, continuous evolution of drug-resistant bacteria leads to the emergence of new $\mathrm{M} \beta \mathrm{L}$ variants, which makes the situation more discouraging [45]. In our study, an SVM model of IMP-1 inhibitors and putative noninhibitors was established based on the 24 SDS-SVM selected descriptors and validated by fivefold cross-validation and an independent test set. Then the established SVM classification model combined with docking method were used to virtual screen a merged compound library for new IMP-1 inhibitors. Finally, 25 compounds were selected and 8 were purchased for in vitro IMP-1 inhibitory assays. 4 compounds were found to have $\mathrm{IC}_{50}<300 \mu \mathrm{M}$ and 2 of them have novel scaffolds, which worth further optimization. The 17 commercial unavailable compounds might also have potential inhibitory activity against IMP-1, and the synthetic work is in progress.

This work demonstrates that combined SVM-based virtual screening and docking method considerably increase hit rate and enrichment factor. Most importantly, it is in a timely, low-resource manner compared with the individual methods.
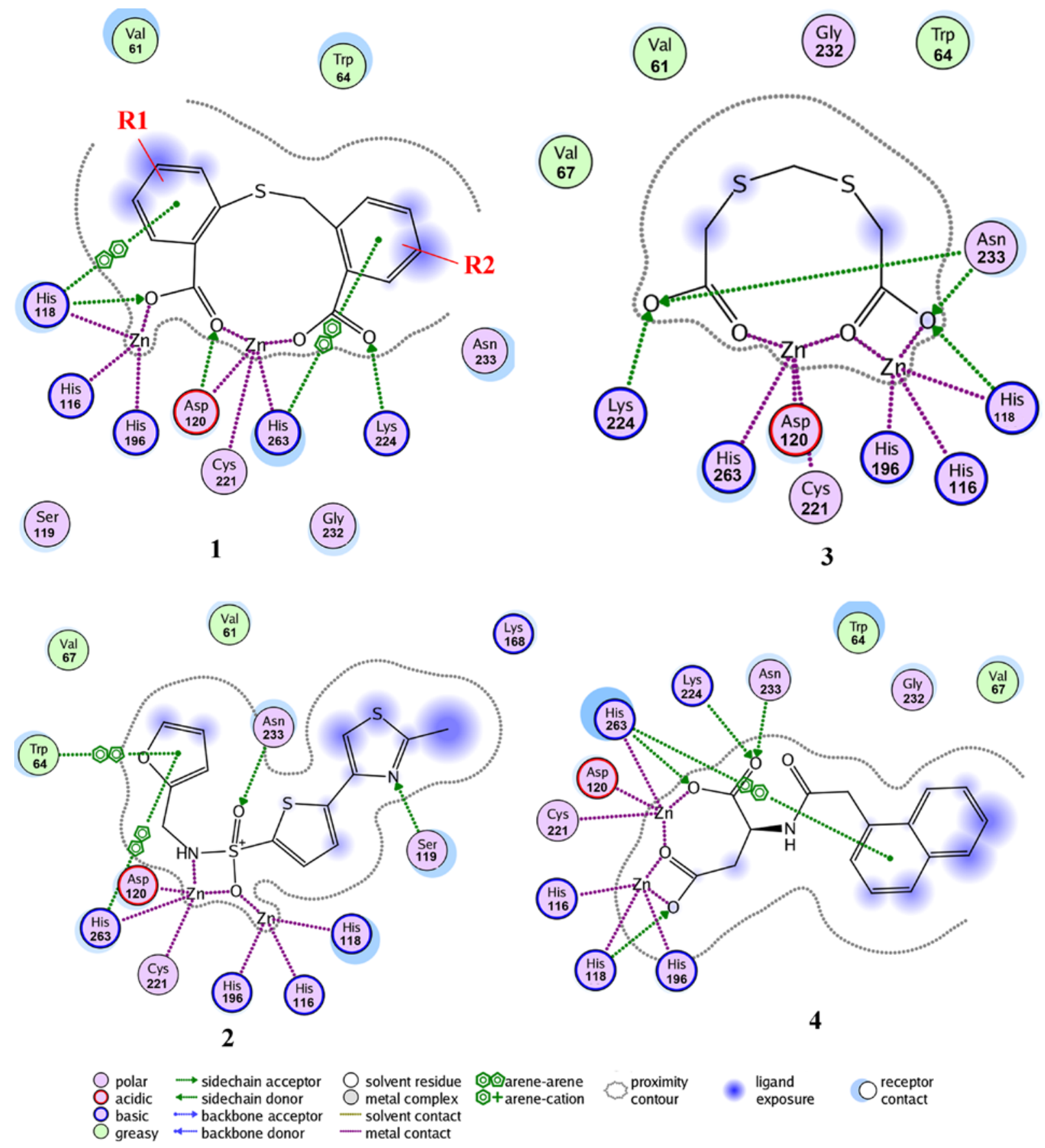

Figure 5. Binding interactions of the active compounds with IMP-1 (PDB code: 1JJE) 


\section{Statement of Competing Interests}

The authors have no competing interests.

\section{Acknowledgments}

This work has been supported by the Fundamental Research Funds for the Central Universities of China (JKZ2009007), the National Natural Science Foundation of China (Grant No. 31300643), and the Priority Academic Program Development of Jiangsu Higher Education Institutions (PAPD).

\section{References}

[1] Ambler, R.P. "The structure of beta-lactamases." Philos Trans $R$ Soc Lond B, 289(1036). 321-331. May. 1980.

[2] Iyobe, S., Kusadokoro, H., Ozaki, J., Matsumura, N., Minami, S., Haruta, S., Sawai, T., O'Hara, K. "Amino acid substitutions in a variant of IMP-1 metallo-beta-lactamase." Antimicrob Agents Chemother, 44 (8). 2023-2027. Aug. 2000.

[3] Toney, J.H., Hammond, G.G., Fitzgerald, P.M., Sharma, N., Balkovec, J.M., Rouen, G.P., Olson, S.H., Hammond, M.L., Greenlee, M.L., Gao, Y.D. "Succinic acids as potent inhibitors of plasmid-borne IMP-1 metallo-beta-lactamase.” J Biol Chem, 276(34). 31913-31918. Jun. 2001.

[4] Siemann, S., Clarke, A.J., Viswanatha, T., Dmitrienko, G.I. "Thiols as classical and slow-binding inhibitors of IMP-1 and other binuclear metallo-beta-lactamases.” Biochemistry, 42(6). 1673-1683. Feb. 2003.

[5] Hammond, G.G., Huber, J.L., Greenlee, M.L., Laub, J.B., Young, K., Silver, L.L., Balkovec, J.M., Pryor, K.D., Wu, J.K., Leiting, B., Pompliano, D.L., Toney, J.H. "Inhibition of IMP-1 metallo-betalactamase and sensitization of IMP-1-producing bacteria by thioester derivatives.” FEMS Microbiol Lett, 179(2). 289-296. Oct. 1999.

[6] Chikauchi, K., Ida, M., Abe, T., Hiraiwa, Y., Morinaka, A., Kudo, T. "Metallo- $\beta$-Lactamase Inhibitors.” US 2008/0090825 A1. Apr. 17, 2008.

[7] Nagano, R., Adachi, Y., Imamura, H., Yamada, K., Hashizume, T., Morishima, H. "Carbapenem derivatives as potential inhibitors of various beta-lactamases, including class B metallo-betalactamases.” Antimicrob Agents Chemother, 43(10). 2497-2503. Oct. 1999.

[8] Olsen, L., Jost, S., Adolph, H.W., Pettersson, I., Hemmingsen, L., Jørgensen, F.S. "New leads of metallo-beta-lactamase inhibitors from structure-based pharmacophore design.” Bioorg Med Chem, 14(8). 2627-2635. Apr. 2006.

[9] Olsen, L., Pettersson, I., Hemmingsen, L., Adolph, H.W., Jørgensen, F.S. "Docking and scoring of metallo-beta-lactamases inhibitors.” J Comput Aid Mol Des, 18(4). 287-302. Apr. 2004.

[10] Oelschlaeger, P., Schmid, R.D., Pleiss, J. "Insight into the mechanism of the IMP-1 metallo-beta-lactamase by molecular dynamics simulations.” Protein Eng, 16(5). 341-350. May. 2003.

[11] Chen, J., Yu, R., Shen, B., Xu, Y., Liu, Y., Zheng, H., Yao, W. "Docking-based 3D-QSAR modeling of the inhibitors of IMP metallo- $\beta$-lactamase.” Med Chem Res, 22(4). 1730-1739. Apr. 2012.

[12] Kitchen, D.B., Decornez, H., Furr, J.R., Bajorath, J. “Docking and scoring in virtual screening for drug discovery: methods and applications.” Nat Rev Drug Discov, 3(11). 935-949. Nov. 2004

[13] $\mathrm{Hu}, \mathrm{X} ., \mathrm{Li}, \mathrm{Q}$. "Using support vector machine to predict beta- and gamma-turns in proteins.” J Comput Chem, 29(12). 1867-1875. Sep. 2008.

[14] Maupetit, J., Derreumaux, P., Tufféry, P. “A fast method for largescale de novo peptide and miniprotein structure prediction." $J$ Comput Chem, 31(4). 726-738. Mar. 2010.

[15] Chang, W.C., Lee, T.Y., Shien, D.M., Hsu, J.B., Horng, J.T., Hsu, P.C., Wang, T.Y., Huang, H.D., Pan, R.L. "Incorporating support vector machine for identifying protein tyrosine sulfation sites." $J$ Comput Chem, 30(15). 2526-2537. Nov. 2009.
[16] Shien, D.M., Lee, T.Y., Chang, W.C., Hsu, J.B., Horng, J.T., Hsu, P.C., Wang, T.Y., Huang, H.D. "Incorporating structural characteristics for identification of protein methylation sites.” $J$ Comput Chem, 30(9). 1532-1543. Jul. 2009.

[17] Lee, T.Y., Hsu, J.B., Lin, F.M., Chang, W.C., Hsu, P.C., Huang, H.D. "N-Ace: using solvent accessibility and physicochemical properties to identify protein $\mathrm{N}$-acetylation sites.” $J$ Comput Chem, 31(15). 2759-2771. Nov. 2010.

[18] Du, H., Wang, J., Zhang, X., Hu, Z. "A novel quantitative structure-activity relationship method to predict the affinities of MT3 melatonin binding site.” Eur J Med Chem, 43(12). 28612869. Dec. 2008.

[19] Ma, X.H., Wang, R., Tan, C.Y., Jiang, Y.Y., Lu, T., Rao, H.B., Li, X.Y., Go, M.L., Low, B.C., Chen, Y.Z. "Virtual screening of selective multitarget kinase inhibitors by combinatorial support vector machines.” Mol Pharm, 7(5). 1545-1560. Oct. 2010.

[20] Yang, X.G., Lv, W., Chen, Y.Z., Xue, Y. "In silico prediction and screening of gamma-secretase inhibitors by molecular descriptors and machine learning methods." J Comput Chem, 31(6). 12491258. Apr. 2010.

[21] Liu, X.H., Song, H.Y., Zhang, J.X., Han, B.C., Wei, X.N., Ma, X.H., Cui, W.K., Chen, Y.Z. "Identifying Novel Type ZBGs and Nonhydroxamate HDAC Inhibitors Through a SVM Based Virtual Screening Approach” Mol Inf, 29(5). 407-420. May. 2010.

[22] Liu, X.H., Song, H.Y., Ma, X.H., Lear, M.J., Chen, Y.Z. "Virtual Screening Prediction of New Potential Organocatalysts for Direct Aldol Reactions.” J Mol Catal A: Chem, 319(1-2). 114-118. Mar. 2010.

[23] Liu, X.H., Ma, X.H., Tan, C.Y., Jiang, Y.Y., Go, M.L., Low, B.C., Chen, Y.Z. "Virtual screening of Abl inhibitors from large compound libraries by support vector machines." J Chem Inf Model, 49(9). 2101-2110. Sep. 2009.

[24] Rao, H., Li, Z., Li, X., Ma, X., Ung, C., Li, H., Liu, X., Chen, Y. "Identification of small molecule aggregators from large compound libraries by support vector machines." J Comput Chem, 31(4), 752-763. Mar. 2010.

[25] Ren, J.X., Li, L.L., Zheng, R.L., Xie, H.Z., Cao, Z.X., Feng, S., Pan, Y.L., Chen, X., Wei, Y.Q., Yang, S.Y. "Discovery of novel Pim-1 kinase inhibitors by a hierarchical multistage virtual screening approach based on SVM model, pharmacophore, and molecular docking.” J Chem Inf Model, 51(6). 1364-1375. Jun. 2011.

[26] Toney, J.H., Cleary, K.A., Hammond, G.G., Yuan, X., May, W.J., Hutchins, S.M., Ashton, W.T., Vanderwall, D.E. "Structureactivity relationships of biphenyl tetrazoles as metallo-betalactamase inhibitors.” Bioorg Med Chem Lett, 9(18). 2741-2746. Sep. 1999.

[27] Payne, D.J., Hueso-Rodriguez, J.A., Boyd, H., Concha, N.O., Janson, C.A., Gilpin, M., Bateson, J.H., Cheever, C., Niconovich, N.L., Pearson, S., Rittenhouse, S., Tew, D., Diez, E., Perez, P., De La Fuente, J., Rees, M., Rivera-Sagredo, A. "Identification of a series of tricyclic natural products as potent broad-spectrum inhibitors of metallo-beta-lactamases." Antimicrob Agents Chemother, 46(6). 1880-1886. Jun. 2002.

[28] Siemann, S., Evanoff, D.P., Marrone, L., Clarke, A.J., Viswanatha, T., Dmitrienko, G.I., "N-arylsulfonyl hydrazones as inhibitors of IMP-1 metallo-beta-lactamase." Antimicrob Agents Chemother, 46(8). 2450-2457. Aug. 2002.

[29] Jin, W., Arakawa, Y., Yasuzawa, H., Taki, T., Hashiguchi, R., Mitsutani, K., Shoga, A., Yamaguchi, Y., Kurosaki, H., Shibata, N., Ohta, M., Goto, M. "Comparative study of the inhibition of metallo-beta-lactamases (IMP-1 and VIM-2) by thiol compounds that contain a hydrophobic group.” Biol Pharm Bull, 27(6). 851856. Jun. 2004.

[30] Kurosaki, H., Yamaguchi, Y., Higashi, T., Soga, K., Matsueda, S., Yumoto, H., Misumi, S., Yamagata, Y., Arakawa, Y., Goto, M. "Irreversible inhibition of metallo-beta-lactamase (IMP-1) by 3(3-mercaptopropionylsulfanyl)propionic acid pentafluorophenyl ester.” Angew Chem Int Ed Engl, 44(25). 3861-3864. Jun. 2005.

[31] Kurosaki, H., Yamaguchi, Y., Yasuzawa, H., Jin, W., Yamagata, Y., Arakawa, Y. "Probing, inhibition, and crystallographic characterization of metallo-beta-lactamase (IMP-1) with fluorescent agents containing dansyl and thiol groups." ChemMedChem, 1(9). 969-972. Sep. 2006.

[32] Horsfall, L.E., Garau, G., Lienard, B.M., Dideberg, O., Schofield, C.J., Frere, J.M., Galleni, M. "Competitive inhibitors of the CphA metallo-beta-lactamase from Aeromonas hydrophila.” Antimicrob Agents Chemother, 51(6). 2136-2142. Jun. 2007. 
[33] Liénard, B.M., Horsfall, L.E., Galleni, M., Frère, J.M., Schofield, C.J. "Inhibitors of the FEZ-1 metallo-beta-lactamase." Bioorg Med Chem Lett, 17(4). 964-968. Feb. 2007.

[34] Liénard, B.M., Garau, G., Horsfall, L., Karsisiotis, A.I., Damblon, C., Lassaux, P., Papamicael, C., Roberts, G.C., Galleni, M., Dideberg, O., Frère, J.M., Schofield, C.J. "Structural basis for the broad-spectrum inhibition of metallo-beta-lactamases by thiols." Org Biomol Chem, 6(13). 2282-2294. Jul. 2008.

[35] Dmitrienko, G.I., Viswanatha, T., Johnson, J.W., Ramadhar, T.R. "Inhibitors of class B and class D $\beta$-lactamases." WO 2009/114921 A1, Sep. 24, 2009.

[36] Hiraiwa, Y., Morinaka, A., Fukushima, T., Kudo, T. "Metallobeta-lactamase inhibitory activity of phthalic acid derivatives." Bioorg Med Chem Lett, 19(17). 5162-5165. Jul. 2009.

[37] Minond, D., Saldanha, S.A., Subramaniam, P., Spaargaren, M., Spicer, T., Fotsing, J.R., Weide, T., Fokin, V.V., Sharpless, K.B., Galleni, M., Bebrone, C., Lassaux, P., Hodder, P. "Inhibitors of VIM-2 by screening pharmacologically active and click-chemistry compound libraries.” Biorg Med Chem, 17(14). 5027-5037. Jul. 2009.

[38] Johnson, J.W., Gretes, M., Goodfellow, V.J., Marrone, L., Heynen, M.L., Strynadka, N.C., Dmitrienko, G.I. "Cyclobutanone analogues of beta-lactams revisited: insights into conformational requirements for inhibition of serine- and metallo-betalactamases.” J Am Chem Soc, 132(8). 2558-2560. Mar. 2010.

[39] Ma, X.H., Wang, R., Yang, S.Y., Li, Z.R., Xue, Y., Wei, Y.C., Low, B.C., Chen, Y.Z. "Evaluation of virtual screening performance of support vector machines trained by sparsely distributed active compounds.” J Chem Inf Model, 48(6). 12271237. Jun. 2008.

[40] Chen, J., Liu, Y., Cheng, T., Lao, X., Gao, X., Zheng, H., Yao, W. "A common binding mode that may facilitate the design of novel broad-spectrum inhibitors against metallo- $\beta$-lactamases.” Med Chem Res, DOI: 10.1007/s00044-013-0646-9. May. 2013.

[41] Jones, G., Willett, P., Glen, R.C., Leach, A.R., Taylor, R. "Development and validation of a genetic algorithm for flexible docking.” J Mol Biol, 267(3). 727-748. Apr. 1997.

[42] Shen, B., Yu, Y., Chen, H., Cao, X., Lao, X., Fang, Y., Shi, Y., Chen, J., Zheng, H. "Inhibitor discovery of full-length New Delhi metallo- $\beta$-lactamase-1 (NDM-1).” PLoS One, 8(5). e62955. May. 2013.

[43] Xie, Q.Q., Zhong, L., Pan, Y.L., Wang, X.Y., Zhou, J.P., Di-Wu, L., Huang, Q., Wang, Y.L., Yang, L.L., Xie, H.Z., Yang, S.Y. "Combined SVM-based and docking-based virtual screening for retrieving novel inhibitors of c-Met.” Eur J Med Chem, 46(9). 3675-3680. Sep. 2011.

[44] Galleni, M., Lamotte-Brasseur, J., Rossolini, G.M., Spencer, J., Dideberg, O., Frere, J.M., Metallo-beta-lactamases Working Group. "Standard numbering scheme for class B beta-lactamases." Antimicrob Agents Chemother, 45(3). 660-663. Mar. 2001.

[45] Chen, J., Shang, X., Hu, F., Lao, X., Gao, X., Zheng, H., Yao, W. "ß-Lactamase Inhibitors: An Update." Mini Rev Med Chem, 13(13).1846-1861. Nov. 2013.

\section{Supplementary Data}

\begin{tabular}{|c|c|c|}
\hline Index & Molecule & $\begin{array}{l}\text { Activity } \\
\text { (IC50 or Ki in } \mu \mathrm{M} \text { ) }\end{array}$ \\
\hline 1 & s1cc $(\mathrm{SC}=2 \mathrm{C}(\mathrm{C} 3 \mathrm{~N}(\mathrm{C}(=\mathrm{O}) \mathrm{C} 3 \mathrm{C}(\mathrm{O}) \mathrm{C}) \mathrm{C}=2 \mathrm{C}(\mathrm{O})=\mathrm{O}) \mathrm{C}) \mathrm{c} 2 \mathrm{c} 1 \mathrm{cccc} 2$ & 0.70 \\
\hline 2 & $\operatorname{s1c} 2 \mathrm{c}(\operatorname{cc} 1 \mathrm{SC}=1 \mathrm{C}(\mathrm{C} 3 \mathrm{~N}(\mathrm{C}(=\mathrm{O}) \mathrm{C} 3 \mathrm{C}(\mathrm{O}) \mathrm{C}) \mathrm{C}=1 \mathrm{C}(\mathrm{OC})=\mathrm{O}) \mathrm{C}) \mathrm{cccc} 2$ & 10 \\
\hline 3 & s1c2c(cc1SCC1NCC $(\mathrm{SC}=3 \mathrm{C}(\mathrm{C} 4 \mathrm{~N}(\mathrm{C}(=\mathrm{O}) \mathrm{C} 4 \mathrm{C}(\mathrm{O}) \mathrm{C}) \mathrm{C}=3 \mathrm{C}(\mathrm{O})=\mathrm{O}) \mathrm{C}) \mathrm{C} 1) \mathrm{cccc} 2$ & 10 \\
\hline 4 & s1c2c(nc1SC $=1 \mathrm{C}(\mathrm{C} 3 \mathrm{~N}(\mathrm{C}(=\mathrm{O}) \mathrm{C} 3 \mathrm{C}(\mathrm{O}) \mathrm{C}) \mathrm{C}=1 \mathrm{C}(\mathrm{O})=\mathrm{O}) \mathrm{C}) \mathrm{cccc} 2$ & 2.40 \\
\hline 5 & $\mathrm{~S}(\mathrm{C}=1 \mathrm{C}(\mathrm{C} 2 \mathrm{~N}(\mathrm{C}(=\mathrm{O}) \mathrm{C} 2 \mathrm{C}(\mathrm{O}) \mathrm{C}) \mathrm{C}=1 \mathrm{C}(\mathrm{O})=\mathrm{O}) \mathrm{C}) \mathrm{c} 1[\mathrm{nH}] \mathrm{c} 2 \mathrm{c}(\mathrm{n} 1) \mathrm{cccc} 2$ & 10 \\
\hline 6 & $\mathrm{~S}(\mathrm{C}(=\mathrm{S}) \mathrm{N} 1 \mathrm{CCC}(=\mathrm{CC} 1) \mathrm{C}[\mathrm{N}+] 12 \mathrm{CC}[\mathrm{N}+](\mathrm{CC} 1)(\mathrm{CC} 2) \mathrm{CC}(=\mathrm{O}) \mathrm{N}) \mathrm{C}=1 \mathrm{C}(\mathrm{C} 2 \mathrm{~N}(\mathrm{C}(=\mathrm{O}) \mathrm{C} 2 \mathrm{C}(\mathrm{O}) \mathrm{C}) \mathrm{C}=1 \mathrm{C}(\mathrm{O})=\mathrm{O}) \mathrm{C}$ & 0.10 \\
\hline 7 & $\mathrm{~S}(\mathrm{C}(=\mathrm{S}) \mathrm{N} 1 \mathrm{CCC}(=\mathrm{CC} 1) \mathrm{C}[\mathrm{N}+] 12 \mathrm{CC}[\mathrm{N}+](\mathrm{CC} 1)(\mathrm{CC} 2) \mathrm{CCO}) \mathrm{C}=1 \mathrm{C}(\mathrm{C} 2 \mathrm{~N}(\mathrm{C}(=\mathrm{O}) \mathrm{C} 2 \mathrm{C}(\mathrm{O}) \mathrm{C}) \mathrm{C}=1 \mathrm{C}(\mathrm{O})=\mathrm{O}) \mathrm{C}$ & 0.1 \\
\hline 8 & $\mathrm{~S}(\mathrm{C}(=\mathrm{S}) \mathrm{N} 1 \mathrm{CCC}(=\mathrm{CC} 1) \mathrm{C}[\mathrm{N}+] 12 \mathrm{CCN}(\mathrm{CC} 1) \mathrm{CC} 2) \mathrm{C}=1 \mathrm{C}(\mathrm{C} 2 \mathrm{~N}(\mathrm{C}(=\mathrm{O}) \mathrm{C} 2 \mathrm{C}(\mathrm{O}) \mathrm{C}) \mathrm{C}=1 \mathrm{C}(\mathrm{O})=\mathrm{O}) \mathrm{C}$ & 0.22 \\
\hline 9 & $\mathrm{~S}(\mathrm{C}(=\mathrm{S}) \mathrm{N} 1 \mathrm{CCC}(=\mathrm{CC} 1) \mathrm{C}[\mathrm{n}+] 1 \mathrm{ccc}(\mathrm{cc} 1) \mathrm{CC}(=\mathrm{O}) \mathrm{N}) \mathrm{C}=1 \mathrm{C}(\mathrm{C} 2 \mathrm{~N}(\mathrm{C}(=\mathrm{O}) \mathrm{C} 2 \mathrm{C}(\mathrm{O}) \mathrm{C}) \mathrm{C}=1 \mathrm{C}(\mathrm{O})=\mathrm{O}) \mathrm{C}$ & 0.22 \\
\hline 10 & $\mathrm{~S}(\mathrm{C}(=\mathrm{S}) \mathrm{N} 1 \mathrm{CCC}=\mathrm{CC} 1) \mathrm{C}=1 \mathrm{C}(\mathrm{C} 2 \mathrm{~N}(\mathrm{C}(=\mathrm{O}) \mathrm{C} 2 \mathrm{C}(\mathrm{O}) \mathrm{C}) \mathrm{C}=1 \mathrm{C}(\mathrm{O})=\mathrm{O}) \mathrm{C}$ & 0.50 \\
\hline 11 & $\mathrm{~S}(\mathrm{C}(=\mathrm{S}) \mathrm{N}(\mathrm{CCO}) \mathrm{C}) \mathrm{C}=1 \mathrm{C}(\mathrm{C} 2 \mathrm{~N}(\mathrm{C}(=\mathrm{O}) \mathrm{C} 2 \mathrm{C}(\mathrm{O}) \mathrm{C}) \mathrm{C}=1 \mathrm{C}(\mathrm{O})=\mathrm{O}) \mathrm{C}$ & 1.40 \\
\hline 12 & $\mathrm{~S}(\mathrm{C}(=\mathrm{S}) \mathrm{N} 1 \mathrm{CCCC} 1) \mathrm{C}=1 \mathrm{C}(\mathrm{C} 2 \mathrm{~N}(\mathrm{C}(=\mathrm{O}) \mathrm{C} 2 \mathrm{C}(\mathrm{O}) \mathrm{C}) \mathrm{C}=1 \mathrm{C}(\mathrm{O})=\mathrm{O}) \mathrm{C}$ & 1.80 \\
\hline 13 & $\mathrm{~S}(\mathrm{C}=1 \mathrm{C}(\mathrm{C} 2 \mathrm{~N}(\mathrm{C}(=\mathrm{O}) \mathrm{C} 2 \mathrm{C}(\mathrm{O}) \mathrm{C}) \mathrm{C}=1 \mathrm{C}(\mathrm{O})=\mathrm{O}) \mathrm{C}) \mathrm{C} 1 \mathrm{CC}(\mathrm{NC} 1) \mathrm{c} 1 \mathrm{cc}(\mathrm{CN}) \mathrm{c}(\mathrm{cc} 1) \mathrm{CN}$ & 0.10 \\
\hline 14 & $\mathrm{~S}(\mathrm{C}=1 \mathrm{C}(\mathrm{C} 2 \mathrm{~N}(\mathrm{C}(=\mathrm{O}) \mathrm{C} 2 \mathrm{C}(\mathrm{O}) \mathrm{C}) \mathrm{C}=1 \mathrm{C}(\mathrm{O})=\mathrm{O}) \mathrm{C}) \mathrm{C} 1 \mathrm{CC}(\mathrm{NC} 1) \mathrm{c} 1 \mathrm{cc}(\mathrm{ccc} 1) \mathrm{CN}$ & 0.40 \\
\hline 15 & $\mathrm{~S}(\mathrm{C}=1 \mathrm{C}(\mathrm{C} 2 \mathrm{~N}(\mathrm{C}(=\mathrm{O}) \mathrm{C} 2 \mathrm{C}(\mathrm{O}) \mathrm{C}) \mathrm{C}=1 \mathrm{C}(\mathrm{O})=\mathrm{O}) \mathrm{C}) \mathrm{C} 1 \mathrm{CC}(\mathrm{NC} 1) \mathrm{c} 1 \mathrm{ccccc} 1$ & 5 \\
\hline 16 & $\mathrm{~S}(\mathrm{C}=1 \mathrm{C}(\mathrm{C} 2 \mathrm{~N}(\mathrm{C}(=\mathrm{O}) \mathrm{C} 2 \mathrm{C}(\mathrm{O}) \mathrm{C}) \mathrm{C}=1 \mathrm{C}(\mathrm{O})=\mathrm{O}) \mathrm{C}) \mathrm{C} 1 \mathrm{CC}(\mathrm{NC} 1) \mathrm{c} 1 \mathrm{ccc}(\mathrm{cc} 1) \mathrm{CNC}$ & 0.70 \\
\hline 17 & $\mathrm{~S}(\mathrm{C}=1 \mathrm{C}(\mathrm{C} 2 \mathrm{~N}(\mathrm{C}(=\mathrm{O}) \mathrm{C} 2 \mathrm{C}(\mathrm{O}) \mathrm{C}) \mathrm{C}=1 \mathrm{C}(\mathrm{O})=\mathrm{O}) \mathrm{C}) \mathrm{C} 1 \mathrm{CNC}(\mathrm{C} 1 \mathrm{C}) \operatorname{c} 1 \mathrm{ccc}(\mathrm{cc} 1) \mathrm{CNC}$ & 10 \\
\hline 18 & $\mathrm{~S}(\mathrm{C}=1 \mathrm{C}(\mathrm{C} 2 \mathrm{~N}(\mathrm{C}(=\mathrm{O}) \mathrm{C} 2 \mathrm{C}(\mathrm{O}) \mathrm{C}) \mathrm{C}=1 \mathrm{C}(\mathrm{O})=\mathrm{O}) \mathrm{C}) \mathrm{C} 1 \mathrm{CC}(\mathrm{N}(\mathrm{C} 1) \mathrm{CCO}) \mathrm{c} 1 \mathrm{ccc}(\mathrm{cc} 1) \mathrm{CN}$ & 0.10 \\
\hline 19 & $\mathrm{~S}(\mathrm{C}=1 \mathrm{C}(\mathrm{C} 2 \mathrm{~N}(\mathrm{C}(=\mathrm{O}) \mathrm{C} 2 \mathrm{C}(\mathrm{O}) \mathrm{C}) \mathrm{C}=1 \mathrm{C}(\mathrm{O})=\mathrm{O}) \mathrm{C}) \mathrm{C} 1 \mathrm{CC}(\mathrm{NC} 1) \mathrm{c} 1 \mathrm{ccc}(\mathrm{cc} 1) \mathrm{CN}$ & 0.40 \\
\hline 20 & $\mathrm{~S}(\mathrm{C}=1 \mathrm{C}(\mathrm{C} 2 \mathrm{~N}(\mathrm{C}(=\mathrm{O}) \mathrm{C} 2 \mathrm{C}(\mathrm{O}) \mathrm{C}) \mathrm{C}=1 \mathrm{C}(\mathrm{O})=\mathrm{O}) \mathrm{C}) \mathrm{C} 1 \mathrm{CC}(\mathrm{NC} 1) \operatorname{cocc}(\operatorname{cc} 1) \mathrm{C}(\mathrm{N}) \mathrm{C}(=\mathrm{O}) \mathrm{N}$ & 5 \\
\hline 21 & $\mathrm{~S}(\mathrm{C}=1 \mathrm{C}(\mathrm{C} 2 \mathrm{~N}(\mathrm{C}(=\mathrm{O}) \mathrm{C} 2 \mathrm{C}(\mathrm{O}) \mathrm{C}) \mathrm{C}=1 \mathrm{C}(\mathrm{O})=\mathrm{O}) \mathrm{C}) \mathrm{C} 1 \mathrm{CC}(\mathrm{NC} 1) \operatorname{c} 1 \mathrm{ccc}(\mathrm{cc} 1) \mathrm{CO}$ & 6.20 \\
\hline 22 & $\mathrm{~S}(\mathrm{C}=1 \mathrm{C}(\mathrm{C} 2 \mathrm{~N}(\mathrm{C}(=\mathrm{O}) \mathrm{C} 2 \mathrm{C}(\mathrm{O}) \mathrm{C}) \mathrm{C}=1 \mathrm{C}(\mathrm{O})=\mathrm{O}) \mathrm{C}) \mathrm{C} 1 \mathrm{CC}(\mathrm{NC} 1) \mathrm{C} 1 \mathrm{CCC}(\mathrm{CC} 1) \mathrm{CN}$ & 9 \\
\hline 23 & $\mathrm{~S}(\mathrm{C}=1 \mathrm{C}(\mathrm{C} 2 \mathrm{~N}(\mathrm{C}(=\mathrm{O}) \mathrm{C} 2 \mathrm{C}(\mathrm{O}) \mathrm{C}) \mathrm{C}=1 \mathrm{C}(\mathrm{O})=\mathrm{O}) \mathrm{C}) \mathrm{C} 1 \mathrm{CC}(\mathrm{NC} 1) \mathrm{Cc} 1 \mathrm{ccc}(\mathrm{cc} 1) \mathrm{CN}$ & 9 \\
\hline 24 & $\mathrm{~S}(\mathrm{CCNCc} 1 \mathrm{ccc}(\mathrm{cc} 1) \mathrm{CN}) \mathrm{C}=1 \mathrm{C}(\mathrm{C} 2 \mathrm{~N}(\mathrm{C}(=\mathrm{O}) \mathrm{C} 2 \mathrm{C}(\mathrm{O}) \mathrm{C}) \mathrm{C}=1 \mathrm{C}(\mathrm{O})=\mathrm{O}) \mathrm{C}$ & 10 \\
\hline 25 & $\operatorname{s1c}(\operatorname{ccc} 1 \mathrm{CN}) \mathrm{C} 1 \mathrm{NCC}(\mathrm{SC}=2 \mathrm{C}(\mathrm{C} 3 \mathrm{~N}(\mathrm{C}(=\mathrm{O}) \mathrm{C} 3 \mathrm{C}(\mathrm{O}) \mathrm{C}) \mathrm{C}=2 \mathrm{C}(\mathrm{O})=\mathrm{O}) \mathrm{C}) \mathrm{C} 1$ & 0.1 \\
\hline 26 & $\operatorname{s1c}(\operatorname{ccc} 1 \mathrm{CNC}) \mathrm{C} 1 \mathrm{NCC}(\mathrm{SC}=2 \mathrm{C}(\mathrm{C} 3 \mathrm{~N}(\mathrm{C}(=\mathrm{O}) \mathrm{C} 3 \mathrm{C}(\mathrm{O}) \mathrm{C}) \mathrm{C}=2 \mathrm{C}(\mathrm{O})=\mathrm{O}) \mathrm{C}) \mathrm{C} 1$ & 0.1 \\
\hline 27 & {$[\mathrm{sH}+] 1 \mathrm{cc}(\mathrm{cc} 1 \mathrm{CNC}) \mathrm{C} 1 \mathrm{NCC}(\mathrm{SC}=2 \mathrm{C}(\mathrm{C} 3 \mathrm{~N}(\mathrm{C}(=\mathrm{O}) \mathrm{C} 3 \mathrm{C}(\mathrm{O}) \mathrm{C}) \mathrm{C}=2 \mathrm{C}(\mathrm{O})=\mathrm{O}) \mathrm{C}) \mathrm{C} 1$} & 0.80 \\
\hline 28 & $\operatorname{s1c} 2 c(\operatorname{cc} 1 S C=1 \mathrm{C}(\mathrm{C} 3 \mathrm{~N}(\mathrm{C}(=\mathrm{O}) \mathrm{C} 3 \mathrm{C}(\mathrm{O}) \mathrm{C}) \mathrm{C}=1 \mathrm{C}(\mathrm{O})=\mathrm{O}) \mathrm{C}) \mathrm{cccc} 2$ & 0.1 \\
\hline 29 & $\mathrm{OC}(=\mathrm{O}) \mathrm{C}=1\left[\mathrm{NH}^{+}\right](\mathrm{N}=\mathrm{C}(\mathrm{CCCC}) \mathrm{C}=1 \mathrm{Cc} 1 \mathrm{ccc}(\mathrm{cc} 1)-\mathrm{c} 1 \mathrm{ccccc} 1-\mathrm{c} 1[\mathrm{nH}] \mathrm{nnn} 1) \mathrm{c} 1 \mathrm{ccccc} 1$ & 200 \\
\hline 30 & Clc1 $1 \operatorname{cccc} 1[N H+] 1 \mathrm{~N}=\mathrm{C}(\mathrm{CCCC}) \mathrm{C}(\mathrm{Cc} 2 \operatorname{ccc}(\operatorname{cc} 2)-\mathrm{c} 2 \operatorname{ccccc} 2-\mathrm{c} 2[\mathrm{nH}] \mathrm{nnn} 2)=\mathrm{C} 1 \mathrm{C}(\mathrm{O})=\mathrm{O}$ & 200 \\
\hline 31 & Clc1cc $([\mathrm{NH}+] 2 \mathrm{~N}=\mathrm{C}(\mathrm{CCCC}) \mathrm{C}(\mathrm{Cc} 3 \operatorname{ccc}(\mathrm{cc} 3)-\mathrm{c} 3 \operatorname{ccccc} 3-\mathrm{c} 3[\mathrm{nH}] \mathrm{nnn} 3)=\mathrm{C} 2 \mathrm{C}(\mathrm{O})=\mathrm{O}) \operatorname{ccc} 1$ & 200 \\
\hline 32 & Clc1ccc $\left(\left[\mathrm{NH}^{+}\right] 2 \mathrm{~N}=\mathrm{C}(\mathrm{CCCC}) \mathrm{C}(\mathrm{Cc} 3 \operatorname{ccc}(\mathrm{cc} 3)-\mathrm{c} 3 \operatorname{ccccc} 3-\mathrm{c} 3[\mathrm{nH}] \mathrm{nnn} 3)=\mathrm{C} 2 \mathrm{C}(\mathrm{O})=\mathrm{O}\right) \mathrm{cc} 1$ & 200 \\
\hline 33 & $\mathrm{Clc1c}([\mathrm{NH}+] 2 \mathrm{~N}=\mathrm{C}(\mathrm{CCCC}) \mathrm{C}(\mathrm{Cc} 3 \operatorname{ccc}(\mathrm{cc} 3)-\mathrm{c} 3 \operatorname{ccccc} 3-\mathrm{c} 3[\mathrm{nH}] \mathrm{nnn} 3)=\mathrm{C} 2 \mathrm{C}(\mathrm{O})=\mathrm{O}) \operatorname{cccc} 1 \mathrm{Cl}$ & 200 \\
\hline 34 & Clc1 $1 \mathrm{cc}(\mathrm{Cl}) \operatorname{ccc} 1[\mathrm{NH}+] 1 \mathrm{~N}=\mathrm{C}(\mathrm{CCCC}) \mathrm{C}(\mathrm{Cc} 2 \operatorname{ccc}(\operatorname{cc} 2)-\mathrm{c} 2 \operatorname{ccccc} 2-\mathrm{c} 2[\mathrm{nH}] \mathrm{nnn} 2)=\mathrm{C} 1 \mathrm{C}(\mathrm{O})=\mathrm{O}$ & 200 \\
\hline 35 & Clc1 $\operatorname{ccc}(\mathrm{Cl}) \operatorname{cc} 1[\mathrm{NH}+] 1 \mathrm{~N}=\mathrm{C}(\mathrm{CCCC}) \mathrm{C}(\mathrm{Cc} 2 \operatorname{ccc}(\operatorname{cc} 2)-\operatorname{coccccc} 2-\operatorname{co} 2[\mathrm{nH}] \mathrm{nnn} 2)=\mathrm{C} 1 \mathrm{C}(\mathrm{O})=\mathrm{O}$ & 200 \\
\hline 36 & Clc1 $1 \operatorname{ccc}(\mathrm{Cl}) \mathrm{c} 1[\mathrm{NH}+] 1 \mathrm{~N}=\mathrm{C}(\mathrm{CCCC}) \mathrm{C}(\mathrm{Cc} 2 \operatorname{ccc}(\operatorname{cc} 2)-\operatorname{coccccc} 2-\mathrm{c} 2[\mathrm{nH}] \mathrm{nnn} 2)=\mathrm{C} 1 \mathrm{C}(\mathrm{O})=\mathrm{O}$ & 200 \\
\hline 37 & Clc1cc $(\mathrm{Cl}) \operatorname{cc}(\mathrm{Cl}) \mathrm{c} 1[\mathrm{NH}+] 1 \mathrm{~N}=\mathrm{C}(\mathrm{CCCC}) \mathrm{C}(\mathrm{Cc} 2 \operatorname{ccc}(\operatorname{cc} 2)-\mathrm{c} 2 \operatorname{ccccc} 2-\mathrm{c} 2[\mathrm{nH}] \mathrm{nnn} 2)=\mathrm{C} 1 \mathrm{C}(\mathrm{O})=\mathrm{O}$ & 200 \\
\hline 38 & $\mathrm{OC}(=\mathrm{O}) \mathrm{C}=1[\mathrm{NH}+](\mathrm{N}=\mathrm{C}(\mathrm{CCCC}) \mathrm{C}=1 \mathrm{Cc} 1 \mathrm{ccc}(\mathrm{cc} 1)-\mathrm{c} 1 \mathrm{ccccc} 1-\mathrm{c} 1[\mathrm{nH}] \mathrm{nnn} 1) \mathrm{c} 1 \mathrm{ccccc} 1 \mathrm{C}$ & 200 \\
\hline 39 & $\mathrm{FC}(\mathrm{F})(\mathrm{F}) \mathrm{c} 1 \mathrm{ccccc} 1[\mathrm{NH}+] 1 \mathrm{~N}=\mathrm{C}(\mathrm{CCCC}) \mathrm{C}(\mathrm{Cc} 2 \operatorname{ccc}(\mathrm{cc} 2)-\mathrm{c} 2 \operatorname{ccccc} 2-\mathrm{c} 2[\mathrm{nH}] \mathrm{nnn} 2)=\mathrm{C} 1 \mathrm{C}(\mathrm{O})=\mathrm{O}$ & 200 \\
\hline 40 & $\mathrm{OC}(=\mathrm{O}) \mathrm{C}=1[\mathrm{NH}+](\mathrm{N}=\mathrm{C}(\mathrm{CCCC}) \mathrm{C}=1 \mathrm{Cc} 1 \mathrm{ccc}(\mathrm{cc} 1)-\mathrm{c} 1 \mathrm{ccccc} 1-\mathrm{c} 1[\mathrm{nH}] \mathrm{nnn} 1) \mathrm{c} 1 \mathrm{ccccc} 1[\mathrm{~N}+](=\mathrm{O})[\mathrm{O}-]$ & 200 \\
\hline 41 & $\mathrm{OC}(=\mathrm{O}) \mathrm{C}=1[\mathrm{NH}+](\mathrm{N}=\mathrm{C}(\mathrm{CCCC}) \mathrm{C}=1 \mathrm{Cc} 1 \mathrm{ccc}(\mathrm{cc} 1)-\mathrm{c} 1 \mathrm{ccccc} 1-\mathrm{c} 1[\mathrm{nH}] \mathrm{nnn} 1) \mathrm{c} 1 \mathrm{ccccc} 1-\mathrm{c} 1 \mathrm{ccccc} 1$ & 65 \\
\hline
\end{tabular}




\begin{tabular}{|c|c|c|}
\hline 42 & $\mathrm{O}(\mathrm{C}(=\mathrm{O}) \mathrm{C}=1[\mathrm{NH}+](\mathrm{N}=\mathrm{C}(\mathrm{CCCC}) \mathrm{C}=1 \mathrm{Cc} 1 \mathrm{ccc}(\mathrm{cc} 1)-\mathrm{c} 1 \mathrm{ccccc} 1-\mathrm{c} 1[\mathrm{nH}] \mathrm{nnn} 1) \mathrm{c} 1 \mathrm{ccccc} 1) \mathrm{CC}$ & 200 \\
\hline 43 & Clc1 $1 \operatorname{ccccc} 1[N H+] 1 \mathrm{~N}=\mathrm{C}(\mathrm{CCC}) \mathrm{C}(\mathrm{Cc} 2 \operatorname{ccc}(\mathrm{cc} 2)-\mathrm{c} 2 \operatorname{ccccc} 2-\mathrm{c} 2[\mathrm{nH}] \mathrm{nnn} 2)=\mathrm{C} 1 \mathrm{C}(\mathrm{OCC})=\mathrm{O}$ & 200 \\
\hline 44 & Clc1cc $([\mathrm{NH}+] 2 \mathrm{~N}=\mathrm{C}(\mathrm{CCCC}) \mathrm{C}(\mathrm{Cc} 3 \operatorname{ccc}(\mathrm{cc} 3)-\mathrm{c} 3 \operatorname{ccccc} 3-\mathrm{c} 3[\mathrm{nH}] \mathrm{nnn} 3)=\mathrm{C} 2 \mathrm{C}(\mathrm{OCC})=\mathrm{O}) \mathrm{ccc} 1$ & 76 \\
\hline 45 & $\mathrm{Clc} 1 \mathrm{ccc}([\mathrm{NH}+] 2 \mathrm{~N}=\mathrm{C}(\mathrm{CCCC}) \mathrm{C}(\mathrm{Cc} 3 \operatorname{ccc}(\operatorname{cc} 3)-\mathrm{c} 3 \operatorname{ccccc} 3-\mathrm{c} 3[\mathrm{nH}] \mathrm{nnn} 3)=\mathrm{C} 2 \mathrm{C}(\mathrm{OCC})=\mathrm{O}) \mathrm{cc} 1$ & 70 \\
\hline 46 & $\mathrm{Clc} 1 \mathrm{c}([\mathrm{NH}+] 2 \mathrm{~N}=\mathrm{C}(\mathrm{CCCC}) \mathrm{C}(\mathrm{Cc} 3 \operatorname{ccc}(\mathrm{cc} 3)-\mathrm{c} 3 \operatorname{ccccc} 3-\mathrm{c} 3[\mathrm{nH}] \mathrm{nnn} 3)=\mathrm{C} 2 \mathrm{C}(\mathrm{OCC})=\mathrm{O}) \operatorname{cccc} 1 \mathrm{Cl}$ & 175 \\
\hline 47 & $\mathrm{Clc} 1 \operatorname{cc}(\mathrm{Cl}) \operatorname{ccc} 1[\mathrm{NH}+] 1 \mathrm{~N}=\mathrm{C}(\mathrm{CCCC}) \mathrm{C}(\mathrm{Cc} 2 \operatorname{ccc}(\operatorname{cc} 2)-\operatorname{coccccc} 2-\mathrm{c} 2[\mathrm{nH}] \mathrm{nnn} 2)=\mathrm{C} 1 \mathrm{C}(\mathrm{OCC})=\mathrm{O}$ & 66 \\
\hline 48 & $\mathrm{Clc} 1 \operatorname{ccc}(\mathrm{Cl}) \operatorname{cc} 1[\mathrm{NH}+] 1 \mathrm{~N}=\mathrm{C}(\mathrm{CCCC}) \mathrm{C}(\mathrm{Cc} 2 \operatorname{ccc}(\operatorname{cc} 2)-\operatorname{cocccc} 2-\mathrm{c} 2[\mathrm{nH}] \mathrm{nnn} 2)=\mathrm{C} 1 \mathrm{C}(\mathrm{OCC})=\mathrm{O}$ & 60 \\
\hline 49 & $\mathrm{Clc} 1 \mathrm{cc}(\mathrm{Cl}) \operatorname{cc}(\mathrm{Cl}) \mathrm{c} 1[\mathrm{NH}+] 1 \mathrm{~N}=\mathrm{C}(\mathrm{CCCC}) \mathrm{C}(\mathrm{Cc} 2 \operatorname{ccc}(\mathrm{cc} 2)-\mathrm{c} 2 \operatorname{ccccc} 2-\mathrm{c} 2[\mathrm{nH}] \mathrm{nnn} 2)=\mathrm{C} 1 \mathrm{C}(\mathrm{OCC})=\mathrm{O}$ & 200 \\
\hline 50 & $\mathrm{O}(\mathrm{C}(=\mathrm{O}) \mathrm{C}=1[\mathrm{NH}+](\mathrm{N}=\mathrm{C}(\mathrm{CCCC}) \mathrm{C}=1 \mathrm{Cc} 1 \mathrm{ccc}(\mathrm{cc} 1)-\mathrm{c} 1 \mathrm{ccccc} 1-\mathrm{c} 1[\mathrm{nH}] \mathrm{nnn} 1) \mathrm{c} 1 \mathrm{ccccc} 1 \mathrm{C}) \mathrm{CC}$ & 200 \\
\hline 51 & $\mathrm{FC}(\mathrm{F})(\mathrm{F}) \mathrm{c} 1 \mathrm{ccccc} 1[\mathrm{NH}+] 1 \mathrm{~N}=\mathrm{C}(\mathrm{CCCC}) \mathrm{C}(\mathrm{Cc} 2 \operatorname{ccc}(\mathrm{cc} 2)-\mathrm{c} 2 \operatorname{ccccc} 2-\mathrm{c} 2[\mathrm{nH}] \mathrm{nn} 2)=\mathrm{C} 1 \mathrm{C}(\mathrm{OCC})=\mathrm{O}$ & 200 \\
\hline 52 & $\mathrm{O}(\mathrm{C}(=\mathrm{O}) \mathrm{C}=1[\mathrm{NH}+](\mathrm{N}=\mathrm{C}(\mathrm{CCCC}) \mathrm{C}=1 \mathrm{Cc} 1 \operatorname{ccc}(\operatorname{cc} 1)-\operatorname{coccccc} 1-\mathrm{c} 1[\mathrm{nH}] \mathrm{nnn} 1) \operatorname{cocccc} 1[\mathrm{~N}+](=\mathrm{O})[\mathrm{O}-]) \mathrm{CC}$ & 200 \\
\hline 53 & $\mathrm{O}(\mathrm{C}(=\mathrm{O}) \mathrm{C}=1[\mathrm{NH}+](\mathrm{N}=\mathrm{C}(\mathrm{CCCC}) \mathrm{C}=1 \mathrm{Cc} 1 \mathrm{ccc}(\mathrm{cc} 1)-\mathrm{c} 1 \mathrm{ccccc} 1-\mathrm{c} 1[\mathrm{nH}] \mathrm{nnn} 1) \mathrm{c} 1 \mathrm{ccccc} 1-\mathrm{c} 1 \mathrm{ccccc} 1) \mathrm{CC}$ & 150 \\
\hline 54 & $\mathrm{~S}(\mathrm{C}(\mathrm{C} \operatorname{1} \operatorname{ccccc} 1) \mathrm{C}(\mathrm{O})=\mathrm{O}) \mathrm{C}(=\mathrm{O}) \mathrm{C}$ & 740 \\
\hline 55 & $\mathrm{~s}(\mathrm{C}(\mathrm{Cc} 1 \mathrm{cc} 2 \mathrm{c} 3 \mathrm{c}(\mathrm{oc} 2 \mathrm{cc} 1) \operatorname{ccc} 3) \mathrm{C}(\mathrm{O})=\mathrm{O}) \mathrm{C}(=\mathrm{O}) \mathrm{C}$ & 160 \\
\hline 56 & $\mathrm{~S}(\mathrm{C}(\mathrm{Cc} 1 \mathrm{ccc}(\mathrm{cc} 1)-\operatorname{c1} 1 \operatorname{ccccc} 1) \mathrm{C}(\mathrm{O})=\mathrm{O}) \mathrm{C}(=\mathrm{O}) \mathrm{C}$ & 74 \\
\hline 57 & $\mathrm{~S}(\mathrm{C}(=\mathrm{O}) \operatorname{c} 1 \operatorname{ccccc} 1) \mathrm{C}(\mathrm{C} 1 \operatorname{ccccc} 1) \mathrm{C}(\mathrm{O})=\mathrm{O}$ & 2.5 \\
\hline 58 & $\mathrm{~S}(\mathrm{C}(=\mathrm{O}) \mathrm{c} 1 \mathrm{cccc} 1) \mathrm{C}(\mathrm{Cc} 1 \mathrm{cc} 2 \mathrm{c} 3 \mathrm{c}($ oc2 $2 \mathrm{cc} 1) \operatorname{cccc} 3) \mathrm{C}(\mathrm{O})=\mathrm{O}$ & 49 \\
\hline 59 & $\mathrm{~S}(\mathrm{C}(=\mathrm{O}) \operatorname{c} 1 \operatorname{ccccc} 1) \mathrm{C}(\mathrm{Cc} 1 \operatorname{ccc}(\operatorname{cc} 1)-\operatorname{c} 1 \operatorname{cccc} 1) \mathrm{C}(\mathrm{O})=\mathrm{O}$ & 120 \\
\hline 60 & $\mathrm{SC}(\mathrm{Cc} 1 \operatorname{ccccc} 1) \mathrm{C}(\mathrm{O})=\mathrm{O}$ & 3.7 \\
\hline 61 & $\operatorname{sC}(\mathrm{Cc} 1 \mathrm{cc} 2 \mathrm{c} 3 \mathrm{c}(\mathrm{oc} 2 \mathrm{cc} 1) \operatorname{cccc} 3) \mathrm{C}(\mathrm{O})=\mathrm{O}$ & 0.25 \\
\hline 62 & $\operatorname{sC}(\mathrm{Cc} 1 \operatorname{ccc}(\operatorname{cc} 1)-\operatorname{c} 1 \operatorname{ccccc} 1) \mathrm{C}(\mathrm{O})=\mathrm{O}$ & 1.2 \\
\hline 63 & $\mathrm{~S}(\mathrm{C}(=\mathrm{O}) \mathrm{C}(\mathrm{NC}(=\mathrm{O}) \mathrm{c} 1 \operatorname{ccccc} 1) \mathrm{C}) \mathrm{CC}(\mathrm{O})=\mathrm{O}$ & 240 \\
\hline 64 & $\mathrm{~S}(\mathrm{C}(\mathrm{Cc} 1 \operatorname{ccc}(\operatorname{cc} 1)-\operatorname{c} 1 \operatorname{ccccc} 1) \mathrm{C}(\mathrm{O})=\mathrm{O}) \mathrm{C}(=\mathrm{O}) \mathrm{C}(\mathrm{NC}(=\mathrm{O}) \mathrm{CNC}(=\mathrm{O}) \operatorname{c} 1 \operatorname{ccccc} 1) \mathrm{C}$ & 50 \\
\hline 65 & $\mathrm{~S}(\mathrm{C}(\mathrm{Cc} 1 \mathrm{ccc}(\mathrm{cc} 1)-\mathrm{c} 1 \mathrm{ccccc} 1) \mathrm{C}(\mathrm{O})=\mathrm{O}) \mathrm{C}(=\mathrm{O}) \mathrm{C}(\mathrm{NC}(=\mathrm{O}) \mathrm{Cc} 1 \mathrm{ccc}(\mathrm{O}) \mathrm{cc} 1) \mathrm{C}$ & 3.10 \\
\hline 66 & $\mathrm{~S}(\mathrm{C}(\mathrm{Cc} 1 \operatorname{ccc}(\mathrm{cc} 1)-\mathrm{c} 1 \mathrm{ccccc} 1) \mathrm{C}(\mathrm{O})=\mathrm{O}) \mathrm{C}(=\mathrm{O}) \mathrm{C}(\mathrm{NC}(=\mathrm{O}) \mathrm{Cc} 1 \mathrm{ccc}(\mathrm{OC}) \mathrm{cc} 1) \mathrm{C}$ & 12 \\
\hline 67 & $\mathrm{~S}(\mathrm{C}(\mathrm{Cc} 1 \operatorname{ccc}(\operatorname{cc} 1)-\operatorname{c} 1 \operatorname{ccccc} 1) \mathrm{C}(\mathrm{O})=\mathrm{O}) \mathrm{C}(=\mathrm{O}) \mathrm{C}(\mathrm{NC}(=\mathrm{O}) \mathrm{Cc} 1 \mathrm{ccc}(\mathrm{N}(\mathrm{C}) \mathrm{C}) \mathrm{cc} 1) \mathrm{C}$ & 55 \\
\hline 68 & 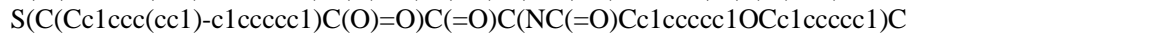 & 24 \\
\hline 69 & $\mathrm{~S}(\mathrm{C}(\mathrm{Cc} 1 \mathrm{ccc}(\mathrm{cc} 1)-\mathrm{c} 1 \operatorname{ccccc} 1) \mathrm{C}(\mathrm{O})=\mathrm{O}) \mathrm{C}(=\mathrm{O}) \mathrm{C}(\mathrm{NC}(=\mathrm{O}) \mathrm{Cc} 1 \mathrm{cccnc} 1) \mathrm{C}$ & 32 \\
\hline 70 & $\mathrm{~S}(\mathrm{C}(\mathrm{Cc} 1 \mathrm{ccc}(\mathrm{cc} 1)-\mathrm{c} 1 \operatorname{ccccc} 1) \mathrm{C}(\mathrm{O})=\mathrm{O}) \mathrm{C}(=\mathrm{O}) \mathrm{C}(\mathrm{NC}(=\mathrm{O}) \mathrm{Cc} 1 \mathrm{c} 2 \mathrm{c}(\operatorname{ccc} 1) \operatorname{cccc} 2) \mathrm{C}$ & 12 \\
\hline 71 & $\mathrm{~S}(\mathrm{C}(\mathrm{Cc} 1 \operatorname{ccc}(\operatorname{cc} 1)-\operatorname{cc} 1 \operatorname{cccc} 1) \mathrm{C}(\mathrm{O})=\mathrm{O}) \mathrm{C}(=\mathrm{O}) \mathrm{C}(\mathrm{NC}(=\mathrm{O}) \operatorname{c1} 1 \mathrm{ccc}(\mathrm{OC}) \mathrm{cc} 1) \mathrm{C}$ & 29 \\
\hline 72 & $\mathrm{~S}(\mathrm{C}(\mathrm{Cc} 1 \mathrm{ccc}(\mathrm{cc} 1)-\mathrm{c} 1 \operatorname{ccccc} 1) \mathrm{C}(\mathrm{O})=\mathrm{O}) \mathrm{C}(=\mathrm{O}) \mathrm{C}(\mathrm{NC}(=\mathrm{O}) \operatorname{c1cc}(\mathrm{OC}) \operatorname{ccc} 1) \mathrm{C}$ & 38 \\
\hline 73 & $\mathrm{~S}(\mathrm{C}(\mathrm{Cc} 1 \operatorname{ccc}(\operatorname{cc} 1)-\operatorname{cocccc} 1) \mathrm{C}(\mathrm{O})=\mathrm{O}) \mathrm{C}(=\mathrm{O}) \mathrm{C}(\mathrm{NC}(=\mathrm{O}) \operatorname{c} 1 \mathrm{cc}(\mathrm{N}(\mathrm{C}) \mathrm{C}) \operatorname{ccc} 1) \mathrm{C}$ & 15 \\
\hline 74 & $\mathrm{~S}(\mathrm{C}(\mathrm{Cc} 1 \mathrm{ccc}(\mathrm{cc} 1)-\mathrm{c} 1 \mathrm{ccccc} 1) \mathrm{C}(\mathrm{O})=\mathrm{O}) \mathrm{C}(=\mathrm{O}) \mathrm{C}(\mathrm{NC}(=\mathrm{O}) \mathrm{c} 1 \mathrm{c}(\mathrm{OC}) \operatorname{cc}(\mathrm{OC}) \mathrm{cc} 1 \mathrm{OC}) \mathrm{C}$ & 46 \\
\hline 75 & $\mathrm{~s}(\mathrm{C}(\mathrm{Cc} 1 \mathrm{ccc}(\mathrm{cc} 1)-\mathrm{c} 1 \mathrm{ccccc} 1) \mathrm{C}(\mathrm{O})=\mathrm{O}) \mathrm{C}(=\mathrm{O}) \mathrm{C}(\mathrm{NC}(=\mathrm{O}) \mathrm{c} 1 \mathrm{cc} 2 \mathrm{c}(\mathrm{cc} 1) \operatorname{cccc} 2) \mathrm{C}$ & 18 \\
\hline 76 & $\mathrm{~S}(\mathrm{C}(\mathrm{Cc} 1 \mathrm{ccccc} 1) \mathrm{C}(\mathrm{O})=\mathrm{O}) \mathrm{C}(=\mathrm{O}) \mathrm{C}(\mathrm{NC}(=\mathrm{O}) \mathrm{C}) \mathrm{C}$ & 63 \\
\hline 77 & $\mathrm{~S}(\mathrm{C}(\mathrm{Cc} 1 \operatorname{ccccc} 1) \mathrm{C}(\mathrm{O})=\mathrm{O}) \mathrm{C}(=\mathrm{O}) \mathrm{C}(\mathrm{NC}(\mathrm{OCC}=\mathrm{C})=\mathrm{O}) \mathrm{C}$ & 64 \\
\hline 78 & $\mathrm{~S}(\mathrm{C}(\mathrm{Cc} 1 \operatorname{ccccc} 1) \mathrm{C}(\mathrm{O})=\mathrm{O}) \mathrm{C}(=\mathrm{O}) \mathrm{C}(\mathrm{NC}(=\mathrm{O}) \mathrm{c} 1 \operatorname{ccccc} 1) \mathrm{C}$ & 20 \\
\hline 79 & $\mathrm{~S}(\mathrm{C}(\mathrm{Cc} 1 \mathrm{ccc}(\mathrm{cc} 1)-\mathrm{c} 1 \operatorname{ccccc} 1) \mathrm{C}(\mathrm{O})=\mathrm{O}) \mathrm{C}(=\mathrm{O}) \mathrm{C}(\mathrm{NC}(=\mathrm{O}) \mathrm{C}) \mathrm{C}$ & 2 \\
\hline 80 & $\mathrm{~s}\left(\mathrm{C}\left(\mathrm{Cc} 1 \mathrm{cc} 2 \operatorname{oc}^{2} \mathrm{c}(\mathrm{c} 2 \mathrm{cc} 1) \operatorname{cccc} 3\right) \mathrm{C}(\mathrm{O})=\mathrm{O}\right) \mathrm{C}(=\mathrm{O}) \mathrm{C}(\mathrm{NC}(\mathrm{OCC}=\mathrm{C})=\mathrm{O}) \mathrm{C}$ & 2.90 \\
\hline 81 & $\mathrm{~S}\left(\mathrm{C}\left(\mathrm{Cc} 1 \mathrm{cc} 2 \mathrm{oc}^{2} \mathrm{c}(\mathrm{c} 2 \mathrm{cc} 1) \operatorname{ccc} 3\right) \mathrm{C}(\mathrm{O})=\mathrm{O}\right) \mathrm{C}(=\mathrm{O}) \mathrm{C}(\mathrm{NC}(=\mathrm{O}) \mathrm{c} 1 \mathrm{ccccc} 1) \mathrm{C}$ & 1.5 \\
\hline 82 & $\mathrm{~s}(\mathrm{C}(\mathrm{Cc} 1 \mathrm{cc} 2 \mathrm{c} 3 \mathrm{c}(\mathrm{oc} 2 \mathrm{cc} 1) \operatorname{ccc} 3) \mathrm{C}(\mathrm{O})=\mathrm{O}) \mathrm{C}(=\mathrm{O}) \mathrm{C}(\mathrm{NC}(=\mathrm{O}) \mathrm{c} 1 \mathrm{ccccc} 1) \mathrm{C}$ & 430 \\
\hline 83 & $\mathrm{~S}(\mathrm{C}(\mathrm{Cc} 1 \mathrm{ccc}(\mathrm{cc} 1)-\mathrm{c} 1 \operatorname{ccccc} 1) \mathrm{C}(\mathrm{O})=\mathrm{O}) \mathrm{C}(=\mathrm{O}) \mathrm{C}(\mathrm{NC}(=\mathrm{O}) \mathrm{C}) \mathrm{C}$ & 18 \\
\hline 84 & $\mathrm{~S}(\mathrm{C}(\mathrm{Cc} 1 \operatorname{ccc}(\operatorname{cc} 1)-\operatorname{c} 1 \operatorname{ccccc} 1) \mathrm{C}(\mathrm{O})=\mathrm{O}) \mathrm{C}(\mathrm{C}(\mathrm{NC}(\mathrm{OCC}=\mathrm{C})=\mathrm{C}) \mathrm{C})=\mathrm{C}$ & 4.40 \\
\hline 85 & $\mathrm{~S}(\mathrm{C}(\mathrm{Cc} 1 \mathrm{ccc}(\mathrm{cc} 1)-\mathrm{c} 1 \operatorname{ccccc} 1) \mathrm{C}(\mathrm{O})=\mathrm{O}) \mathrm{C}(=\mathrm{O}) \mathrm{C}(\mathrm{NC}(=\mathrm{O}) \operatorname{c} 1 \operatorname{ccccc} 1) \mathrm{C}$ & 3.60 \\
\hline 86 & $\mathrm{~S}(\mathrm{C}(\mathrm{Cc} 1 \operatorname{ccc}(\operatorname{cc} 1)-\operatorname{c} 1 \operatorname{cccc} 1) \mathrm{C}(\mathrm{O})=\mathrm{O}) \mathrm{C}(=\mathrm{O}) \mathrm{C}(\mathrm{NC}(=\mathrm{O}) \mathrm{CC}) \mathrm{C}$ & 9.5 \\
\hline 87 & $\mathrm{~S}(\mathrm{C}(\mathrm{Cc} 1 \operatorname{ccc}(\mathrm{cc} 1)-\mathrm{c} 1 \operatorname{ccccc} 1) \mathrm{C}(\mathrm{O})=\mathrm{O}) \mathrm{C}(=\mathrm{O}) \mathrm{C}(\mathrm{NC}(=\mathrm{O}) \mathrm{CCC}) \mathrm{C}$ & 9.30 \\
\hline 88 & $\mathrm{~S}(\mathrm{C}(\mathrm{Cc} 1 \mathrm{ccc}(\mathrm{cc} 1)-\mathrm{c} 1 \mathrm{ccccc} 1) \mathrm{C}(\mathrm{O})=\mathrm{O}) \mathrm{C}(=\mathrm{O}) \mathrm{C}(\mathrm{NC}(=\mathrm{O}) \mathrm{CCCC}) \mathrm{C}$ & 4.80 \\
\hline 89 & $\mathrm{~S}(\mathrm{C}(\mathrm{C} \operatorname{c} 1 \operatorname{ccc}(\operatorname{cc} 1)-\operatorname{c} 1 \operatorname{ccccc} 1) \mathrm{C}(\mathrm{O})=\mathrm{O}) \mathrm{C}(=\mathrm{O}) \mathrm{C}(\mathrm{NC}(=\mathrm{O}) \mathrm{CCCC}) \mathrm{C}$ & 13 \\
\hline 90 & $\mathrm{~S}(\mathrm{C}(\mathrm{Cc} 1 \mathrm{ccc}(\mathrm{cc} 1)-\mathrm{c} 1 \mathrm{ccccc} 1) \mathrm{C}(\mathrm{O})=\mathrm{O}) \mathrm{C}(=\mathrm{O}) \mathrm{C}(\mathrm{NC}(=\mathrm{O}) \mathrm{C}(\mathrm{C}) \mathrm{C}) \mathrm{C}$ & 9.70 \\
\hline 91 & $\mathrm{~S}(\mathrm{C}(\mathrm{Cc} 1 \operatorname{ccc}(\operatorname{cc} 1)-\operatorname{c} 1 \operatorname{ccccc} 1) \mathrm{C}(\mathrm{O})=\mathrm{O}) \mathrm{C}(=\mathrm{O}) \mathrm{C}(\mathrm{NC}(=\mathrm{O}) \mathrm{CC}(\mathrm{C}) \mathrm{C}) \mathrm{C}$ & 5.30 \\
\hline 92 & $\mathrm{~S}(\mathrm{C}(\mathrm{Cc} 1 \mathrm{ccc}(\mathrm{cc} 1)-\mathrm{c} 1 \mathrm{ccccc} 1) \mathrm{C}(\mathrm{O})=\mathrm{O}) \mathrm{C}(=\mathrm{O}) \mathrm{C}(\mathrm{NC}(=\mathrm{O}) \mathrm{C}=\mathrm{CC}) \mathrm{C}$ & 33 \\
\hline 93 & $\mathrm{~S}(\mathrm{C}(\mathrm{Cc} 1 \mathrm{ccc}(\operatorname{cc} 1)-\mathrm{c} 1 \operatorname{ccccc} 1) \mathrm{C}(\mathrm{O})=\mathrm{O}) \mathrm{C}(=\mathrm{O}) \mathrm{C}(\mathrm{NC}(=\mathrm{O}) \mathrm{CCC}(\mathrm{O})=\mathrm{O}) \mathrm{C}$ & 4.70 \\
\hline 94 & $\mathrm{~S}(\mathrm{C}(\mathrm{Cc} 1 \mathrm{ccc}(\operatorname{cc} 1)-\operatorname{c} 1 \operatorname{ccccc} 1) \mathrm{C}(\mathrm{O})=\mathrm{O}) \mathrm{C}(=\mathrm{O}) \mathrm{C}(\mathrm{NC}(=\mathrm{O}) \mathrm{CSCC}(\mathrm{O})=\mathrm{O}) \mathrm{C}$ & 13 \\
\hline 95 & $\mathrm{~S}(\mathrm{C}(\mathrm{Cc} 1 \mathrm{ccc}(\operatorname{cc} 1)-\operatorname{c} 1 \operatorname{ccccc} 1) \mathrm{C}(\mathrm{O})=\mathrm{O}) \mathrm{C}(=\mathrm{O}) \mathrm{C}(\mathrm{NC}(=\mathrm{O}) \mathrm{CCCC}(\mathrm{O})=\mathrm{O}) \mathrm{C}$ & 91 \\
\hline 96 & $\mathrm{~S}(\mathrm{C}(\mathrm{Cc} 1 \operatorname{ccc}(\operatorname{cc} 1)-\operatorname{cocccc} 1) \mathrm{C}(\mathrm{O})=\mathrm{O}) \mathrm{C}(=\mathrm{O}) \mathrm{C}(\mathrm{NC}(=\mathrm{O}) \mathrm{Cc} 1 \operatorname{ccccc} 1) \mathrm{C}$ & 5.80 \\
\hline 97 & $\mathrm{~S}(\mathrm{C}(\mathrm{Cc} 1 \mathrm{ccc}(\mathrm{ccc} 1)-\mathrm{c} 1 \operatorname{ccccc} 1) \mathrm{C}(\mathrm{O})=\mathrm{O}) \mathrm{C}(=\mathrm{O}) \mathrm{C}(\mathrm{NC}(=\mathrm{O}) \mathrm{COc} 1 \operatorname{ccccc} 1) \mathrm{C}$ & 1 \\
\hline 98 & $\mathrm{~S}(\mathrm{C}(\mathrm{Cc} 1 \mathrm{ccc}(\mathrm{cc} 1)-\mathrm{c} 1 \operatorname{ccccc} 1) \mathrm{C}(\mathrm{O})=\mathrm{O}) \mathrm{C}(=\mathrm{O}) \mathrm{C}(\mathrm{NC}(=\mathrm{O}) \mathrm{CCc} 1 \operatorname{ccccc} 1) \mathrm{C}$ & 15 \\
\hline 99 & $\mathrm{~S}(\mathrm{C}(\mathrm{Cc} 1 \operatorname{ccc}(\operatorname{cc} 1)-\operatorname{c} 1 \operatorname{ccccc} 1) \mathrm{C}(\mathrm{O})=\mathrm{O}) \mathrm{C}(=\mathrm{O}) \mathrm{C}(\mathrm{NC}(=\mathrm{O}) \mathrm{C}=\mathrm{Cc} 1 \operatorname{ccccc} 1) \mathrm{C}$ & 11 \\
\hline 100 & $\mathrm{~S}(\mathrm{C}(\mathrm{Cc} 1 \operatorname{ccc}(\operatorname{cc} 1)-\operatorname{c} 1 \operatorname{ccccc} 1) \mathrm{C}(\mathrm{O})=\mathrm{O}) \mathrm{C}(=\mathrm{O}) \mathrm{C}(\mathrm{NC}(=\mathrm{O}) \mathrm{CCC}(=\mathrm{O}) \operatorname{c} 1 \operatorname{ccccc} 1) \mathrm{C}$ & 0.25 \\
\hline 101 & $\mathrm{~S}(\mathrm{C}(\mathrm{Cc} 1 \operatorname{ccccc} 1) \mathrm{C}(\mathrm{O})=\mathrm{O}) \mathrm{C}(=\mathrm{O}) \mathrm{C}$ & 12 \\
\hline 102 & $\mathrm{~s}(\mathrm{C}(\mathrm{Cc} 1 \mathrm{cc} 2 \mathrm{c} 3 \mathrm{c}(\mathrm{oc} 2 \mathrm{cc} 1) \operatorname{ccc} 3) \mathrm{C}(\mathrm{O})=\mathrm{O}) \mathrm{C}(=\mathrm{O}) \mathrm{C}$ & 0.045 \\
\hline 103 & $\mathrm{~S}(\mathrm{C}(\mathrm{Cc} 1 \operatorname{ccc}(\operatorname{cc} 1)-\operatorname{c} 1 \operatorname{ccccc} 1) \mathrm{C}(\mathrm{O})=\mathrm{O}) \mathrm{C}(=\mathrm{O}) \mathrm{C}$ & 0.084 \\
\hline 104 & $\mathrm{~S}(\mathrm{C}(=\mathrm{O}) \operatorname{c} 1 \operatorname{cccc} 1) \mathrm{C}(\mathrm{C} 1 \operatorname{ccccc} 1) \mathrm{C}(\mathrm{O})=\mathrm{O}$ & 0.064 \\
\hline 105 & $\mathrm{~S}(\mathrm{C}(=\mathrm{O}) \operatorname{c1ccccc} 1) \mathrm{C}(\mathrm{Cc} 1 \mathrm{cc} 2 \mathrm{c} 3 \mathrm{c}(\mathrm{oc} 2 \mathrm{cc} 1) \operatorname{cccc} 3) \mathrm{C}(\mathrm{O})=\mathrm{O}$ & 0.0013 \\
\hline 106 & $\mathrm{~S}(\mathrm{C}(=\mathrm{O}) \operatorname{c} 1 \operatorname{ccccc} 1) \mathrm{C}(\mathrm{Cc} 1 \operatorname{ccc}(\operatorname{cc} 1)-\operatorname{c} 1 \operatorname{ccccc} 1) \mathrm{C}(\mathrm{O})=\mathrm{O}$ & 0.00041 \\
\hline 107 & $\operatorname{SC}(\mathrm{Cc} 1 \operatorname{ccccc} 1) \mathrm{C}(\mathrm{O})=\mathrm{O}$ & 0.086 \\
\hline 108 & $\mathrm{SC}(\mathrm{Cc} 1 \mathrm{cc} 2 \mathrm{c} 3 \mathrm{c}(\mathrm{oc} 2 \mathrm{cc} 1) \operatorname{cccc} 3) \mathrm{C}(\mathrm{O})=\mathrm{O}$ & 0.023 \\
\hline 109 & $\mathrm{SC}(\mathrm{Cc} 1 \mathrm{ccc}(\mathrm{cc} 1)-\mathrm{c} 1 \operatorname{ccccc} 1) \mathrm{C}(\mathrm{O})=\mathrm{O}$ & 0.057 \\
\hline 110 & O1c2cc $(\operatorname{ccc} 2 \mathrm{OC} 1) \mathrm{CC}(\mathrm{C}(\mathrm{Cc} 1 \mathrm{cc} 2 \mathrm{OCOc} 2 \mathrm{cc} 1) \mathrm{C}(\mathrm{O})=\mathrm{O}) \mathrm{C}(\mathrm{O})=\mathrm{O}$ & 0.009 \\
\hline 111 & $\mathrm{OC}(=\mathrm{O}) \mathrm{C}(\mathrm{Cc} 1 \mathrm{ccccc} 1) \mathrm{C}(\mathrm{Cc} 1 \operatorname{ccccc} 1) \mathrm{C}(\mathrm{O})=\mathrm{O}$ & 0.0027 \\
\hline 112 & $\mathrm{OC}(=\mathrm{O}) \mathrm{C}(\mathrm{Cc} 1 \operatorname{cccc} 1) \mathrm{C}(\mathrm{Cc} 1 \operatorname{ccccc} 1) \mathrm{C}(\mathrm{O})=\mathrm{O}$ & 0.203 \\
\hline 113 & $\mathrm{OC}(=\mathrm{O}) \mathrm{C}(\mathrm{Cc} 1 \mathrm{ccccc} 1) \mathrm{C}(\mathrm{Cc} 1 \operatorname{ccccc} 1) \mathrm{C}(\mathrm{O})=\mathrm{O}$ & 200 \\
\hline 114 & $\mathrm{OC}(=\mathrm{O}) \operatorname{CCC}(\mathrm{O})=\mathrm{O}$ & 6300 \\
\hline 115 & $\mathrm{OC}(=\mathrm{O}) \mathrm{C}(\mathrm{Cc} 1 \operatorname{ccccc} 1) \mathrm{CC}(\mathrm{O})=\mathrm{O}$ & 490 \\
\hline 116 & $\mathrm{OC}(=\mathrm{O}) \mathrm{C}(\mathrm{CC}(\mathrm{O})=\mathrm{O})(\mathrm{C}) \mathrm{C}$ & 10000 \\
\hline 117 & $\mathrm{OC}(=\mathrm{O}) \mathrm{C}(\mathrm{CC}(\mathrm{O})=\mathrm{O})(\mathrm{CC}) \mathrm{C}$ & 10000 \\
\hline 118 & $\mathrm{OC}(=\mathrm{O}) \mathrm{C}(\mathrm{C}(\mathrm{C}) \mathrm{c} 1 \operatorname{ccccc} 1) \mathrm{C}(\mathrm{Cc} 1 \operatorname{ccccc} 1) \mathrm{C}(\mathrm{O})=\mathrm{O}$ & 0.013 \\
\hline 119 & $\mathrm{OC}(=\mathrm{O}) \mathrm{C}(\mathrm{C}(\mathrm{C}) \operatorname{c} 1 \operatorname{ccccc} 1) \mathrm{C}(\mathrm{Cc} 1 \operatorname{ccccc} 1) \mathrm{C}(\mathrm{O})=\mathrm{O}$ & 2.7 \\
\hline 120 & O1c2cc( $\operatorname{ccc} 2 \mathrm{OC} 1) \mathrm{CC}(\mathrm{C}(\mathrm{Cc} 1 \operatorname{ccccc} 1) \mathrm{C}(\mathrm{O})=\mathrm{O}) \mathrm{C}(\mathrm{O})=\mathrm{O}$ & 0.0037 \\
\hline 121 & $\mathrm{O} 1 \mathrm{c} 2 \mathrm{c}(\mathrm{c}(\mathrm{C}(\mathrm{O})=\mathrm{O}) \mathrm{c}(\mathrm{O}) \mathrm{c}(\mathrm{O}) \mathrm{c} 2) \mathrm{C}(=\mathrm{O}) \mathrm{c} 2 \mathrm{c} 1 \mathrm{cc}(\mathrm{nc} 2) \mathrm{C}$ & 151 \\
\hline
\end{tabular}




\begin{tabular}{|c|c|c|}
\hline 122 & $\mathrm{O} 1 \mathrm{C}(\mathrm{CC}=2 \mathrm{Oc} 3 \mathrm{c}(\mathrm{c}(\mathrm{C}(\mathrm{O})=\mathrm{O}) \mathrm{c}(\mathrm{O}) \mathrm{c}(\mathrm{O}) \mathrm{c} 3) \mathrm{C}(=\mathrm{O}) \mathrm{C}=2 \mathrm{C} 1 \mathrm{OC}) \mathrm{C}$ & 113 \\
\hline 123 & $\mathrm{O} 1 \mathrm{C}(=\mathrm{CC}=2 \mathrm{Oc} 3 \mathrm{c}(\mathrm{c}(\mathrm{C}(\mathrm{O})=\mathrm{O}) \mathrm{c}(\mathrm{O}) \mathrm{c}(\mathrm{O}) \mathrm{c} 3) \mathrm{C}(=\mathrm{O}) \mathrm{C}=2 \mathrm{C} 1 \mathrm{OC}) \mathrm{C}$ & 26 \\
\hline 124 & $\mathrm{OC}(=\mathrm{O}) \mathrm{c} 1 \mathrm{nc}(\operatorname{ccc} 1) \mathrm{C}(\mathrm{O})=\mathrm{O}$ & 781 \\
\hline 125 & $\operatorname{SCC}(\mathrm{Cc} 1 \operatorname{ccccc} 1) \mathrm{C}(=\mathrm{O}) \mathrm{NCC}(\mathrm{O})=\mathrm{O}$ & 37 \\
\hline 126 & $\mathrm{~S}(=\mathrm{O})(=\mathrm{O})(\mathrm{NN}=\mathrm{Cc} 1 \mathrm{c} 2 \mathrm{c}(\operatorname{ccc} 1 \mathrm{O}) \operatorname{cccc} 2) \operatorname{c} 1 \operatorname{ccc}(\operatorname{cc} 1) \mathrm{C}$ & 40 \\
\hline 127 & $\mathrm{~S}(=\mathrm{O})(=\mathrm{O})(\mathrm{NN}=\mathrm{Cc} 1 \mathrm{c} 2 \mathrm{c}(\operatorname{ccc} 1 \mathrm{O}) \operatorname{cccc} 2) \operatorname{c} 1 \operatorname{ccc}([\mathrm{N}+](=\mathrm{O})[\mathrm{O}-]) \operatorname{cc} 1$ & 48 \\
\hline 128 & $\mathrm{~S}(=\mathrm{O})(=\mathrm{O})(\mathrm{NN}=\mathrm{Cc} 1 \mathrm{c} 2 \mathrm{c}(\operatorname{ccc} 1 \mathrm{O}) \operatorname{cccc} 2) \operatorname{cocc}(\mathrm{OC}) \mathrm{cc} 1$ & 40 \\
\hline 129 & $\mathrm{~S}(=\mathrm{O})(=\mathrm{O})(\mathrm{NN}=\mathrm{Cc} 1 \mathrm{c} 2 \mathrm{c}(\operatorname{ccc} 1 \mathrm{O}) \operatorname{cccc} 2) \operatorname{c} 1 \mathrm{ccc}(\mathrm{OC}(\mathrm{F})(\mathrm{F}) \mathrm{F}) \mathrm{cc} 1$ & 25 \\
\hline 130 & Clc1ccc $(\mathrm{Cl}) \operatorname{cc} 1 \mathrm{~S}(=\mathrm{O})(=\mathrm{O}) \mathrm{NN}=\mathrm{Cc} 1 \mathrm{c} 2 \mathrm{c}(\operatorname{ccc} 1 \mathrm{O}) \operatorname{cccc} 2$ & 40 \\
\hline 131 & Clc1ccc $(\mathrm{Cl}) \operatorname{cc} 1 \mathrm{~S}(=\mathrm{O})(=\mathrm{O}) \mathrm{NN}=\mathrm{Cc} 1 \mathrm{c} 2 \mathrm{c}(\operatorname{ccc} 1 \mathrm{OC}) \operatorname{cccc} 2$ & 10 \\
\hline 132 & Clc1ccc $(\mathrm{Cl}) \operatorname{cc} 1 \mathrm{~S}(=\mathrm{O})(=\mathrm{O}) \mathrm{NN}=\mathrm{Cc} 1 \mathrm{c} 2 \mathrm{c}(\operatorname{cc} 3 \mathrm{c} 1 \mathrm{cccc} 3) \operatorname{cccc} 2$ & 4.5 \\
\hline 133 & $\mathrm{~S}(=\mathrm{O})(=\mathrm{O})(\mathrm{NN}=\mathrm{Cc} 1 \mathrm{c} 2 \mathrm{c}(\operatorname{ccc} 1 \mathrm{O}) \operatorname{ccc} 2) \operatorname{c} 1 \mathrm{c}(\operatorname{cc}(\operatorname{cc} 1 \mathrm{C}) \mathrm{C}) \mathrm{C}$ & 10 \\
\hline 134 & $\mathrm{~S}(=\mathrm{O})(=\mathrm{O})(\mathrm{NN}=\mathrm{Cc} 1 \mathrm{c} 2 \mathrm{c}(\operatorname{ccc} 1 \mathrm{OC}) \operatorname{cccc} 2) \operatorname{coc}(\operatorname{cc}(\operatorname{cc} 1 \mathrm{C}) \mathrm{C}) \mathrm{C}$ & 4.80 \\
\hline 135 & $\mathrm{~S}(=\mathrm{O})(=\mathrm{O})(\mathrm{NN}=\mathrm{Cc} 1 \mathrm{c} 2 \mathrm{c}(\operatorname{cc} 3 \mathrm{c} 1 \mathrm{ccc} c 3) \operatorname{ccc} 2) \operatorname{c} 1 \mathrm{c}(\operatorname{cc}(\operatorname{cc} 1 \mathrm{C}) \mathrm{C}) \mathrm{C}$ & 1.6 \\
\hline 136 & $\mathrm{~S}(=\mathrm{O})(=\mathrm{O})(\mathrm{NN}=\mathrm{Cc} 1 \mathrm{c} 2 \mathrm{c}(\operatorname{ccc} 1 \mathrm{O}) \operatorname{ccc} 2) \operatorname{coc} 2 \mathrm{c}(\mathrm{cc} 1) \operatorname{cccc} 2$ & 10 \\
\hline 137 & $\mathrm{~S}(=\mathrm{O})(=\mathrm{O})(\mathrm{NN}=\mathrm{Cc} 1 \mathrm{c} 2 \mathrm{c}(\operatorname{ccc} 1 \mathrm{OC}) \operatorname{cccc} 2) \operatorname{c} 1 \mathrm{cc} 2 \mathrm{c}(\mathrm{cc} 1) \operatorname{cccc} 2$ & 4 \\
\hline 138 & $\mathrm{~S}(=\mathrm{O})(=\mathrm{O})(\mathrm{NN}=\mathrm{Cc} 1 \mathrm{c} 2 \mathrm{c}(\mathrm{cc} 3 \mathrm{c} 1 \mathrm{ccc} 3) \operatorname{ccc} 2) \operatorname{c} 1 \mathrm{cc} 2 \mathrm{c}(\mathrm{cc} 1) \operatorname{cccc} 2$ & 1.6 \\
\hline 139 & $\mathrm{~S}(=\mathrm{O})(=\mathrm{O})(\mathrm{NN}=\mathrm{Cc} 1 \mathrm{c} 2 \mathrm{c}(\operatorname{ccc} 1 \mathrm{O}) \operatorname{ccc} 2) \operatorname{coc} \mathrm{c}(\operatorname{ccc} 1) \operatorname{cccc} 2$ & 20 \\
\hline 140 & $\mathrm{~S}(=\mathrm{O})(=\mathrm{O})(\mathrm{NN}=\mathrm{Cc} 1 \mathrm{c} 2 \mathrm{c}(\operatorname{ccc} 1 \mathrm{OC}) \operatorname{cccc} 2) \operatorname{coc} 2 \mathrm{c}(\operatorname{ccc} 1) \operatorname{cccc} 2$ & 6.20 \\
\hline 141 & $\mathrm{~S}(=\mathrm{O})(=\mathrm{O})(\mathrm{NN}=\mathrm{Cc} 1 \mathrm{c} 2 \mathrm{c}(\operatorname{cc} 3 \mathrm{c} 1 \mathrm{ccc} 3) \operatorname{ccc} 2) \mathrm{c} 1 \mathrm{c} 2 \mathrm{c}(\operatorname{ccc} 1) \operatorname{cccc} 2$ & 2.40 \\
\hline 142 & $\mathrm{~S}(=\mathrm{O})(=\mathrm{O})(\mathrm{NN}=\mathrm{Cc} 1 \mathrm{c} 2 \mathrm{c}(\operatorname{ccc} 1 \mathrm{O}) \operatorname{cccc} 2) \operatorname{c} 1 \mathrm{ccc}(\operatorname{cc} 1) \mathrm{C}(\mathrm{C})(\mathrm{C}) \mathrm{C}$ & 16 \\
\hline 143 & $\mathrm{~S}(=\mathrm{O})(=\mathrm{O})(\mathrm{NN}=\mathrm{Cc} 1 \mathrm{c} 2 \mathrm{c}(\operatorname{ccc} 1 \mathrm{OC}) \operatorname{cccc} 2) \operatorname{coccc}(\operatorname{cc} 1) \mathrm{C}(\mathrm{C})(\mathrm{C}) \mathrm{C}$ & 3.8 \\
\hline 144 & $\mathrm{~S}(=\mathrm{O})(=\mathrm{O})(\mathrm{NN}=\mathrm{Cc} 1 \mathrm{c} 2 \mathrm{c}(\operatorname{cc} 3 \mathrm{c} 1 \mathrm{ccc} 3) \operatorname{ccc} 2) \operatorname{cocc}(\operatorname{cc} 1) \mathrm{C}(\mathrm{C})(\mathrm{C}) \mathrm{C}$ & 2.2 \\
\hline 145 & $\operatorname{Ic} 1 \operatorname{ccc}(\mathrm{S}(=\mathrm{O})(=\mathrm{O}) \mathrm{NN}=\mathrm{Cc} 2 \mathrm{c} 3 \mathrm{c}(\operatorname{ccc} 2 \mathrm{O}) \operatorname{ccc} 3) \operatorname{cc} 1$ & 17.5 \\
\hline 146 & $\operatorname{Ic} 1 \operatorname{ccc}(\mathrm{S}(=\mathrm{O})(=\mathrm{O}) \mathrm{NN}=\mathrm{Cc} 2 \mathrm{c} 3 \mathrm{c}(\operatorname{ccc} 2 \mathrm{OC}) \operatorname{cccc} 3) \operatorname{cc} 1$ & 6.30 \\
\hline 147 & Ic1 $1 \operatorname{ccc}(\mathrm{S}(=\mathrm{O})(=\mathrm{O}) \mathrm{NN}=\mathrm{Cc} 2 \mathrm{c} 3 \mathrm{c}(\operatorname{cc} 4 \mathrm{c} 2 \operatorname{ccc} 4) \operatorname{cccc} 3) \operatorname{cc} 1$ & 3 \\
\hline 148 & $\operatorname{Ic} 1 \operatorname{ccc}(S(=O)(=O) N N=\operatorname{Cc} 2 c 3 c(\operatorname{ccc} 2) \operatorname{cccc} 3) \operatorname{cc} 1$ & 10.5 \\
\hline 149 & $\operatorname{Ic} 1 \operatorname{ccc}(\mathrm{S}(=\mathrm{O})(=\mathrm{O}) \mathrm{NN}=\mathrm{Cc} 2 \operatorname{ccccc} 2 \mathrm{O}) \operatorname{cc} 1$ & 250 \\
\hline 150 & $\operatorname{Brc} 1 \operatorname{ccc}(\mathrm{S}(=\mathrm{O})(=\mathrm{O}) \mathrm{NN}=\mathrm{Cc} 2 \mathrm{c} 3 \mathrm{c}(\operatorname{ccc} 2 \mathrm{O}) \operatorname{cccc} 3) \operatorname{cc} 1$ & 25 \\
\hline 151 & $\operatorname{Brc} 1 \operatorname{ccc}(\mathrm{S}(=\mathrm{O})(=\mathrm{O}) \mathrm{NN}=\mathrm{Cc} 2 \mathrm{c} 3 \mathrm{c}(\operatorname{ccc} 2 \mathrm{OC}) \operatorname{ccc} 3) \mathrm{cc} 1$ & 13 \\
\hline 152 & $\operatorname{Brc} 1 \operatorname{ccc}(\mathrm{S}(=\mathrm{O})(=\mathrm{O}) \mathrm{NN}=\mathrm{Cc} 2 \mathrm{c} 3 \mathrm{c}(\operatorname{cc} 4 \mathrm{c} 2 \operatorname{cccc} 4) \operatorname{cccc} 3) \operatorname{cc} 1$ & 4.60 \\
\hline 153 & Clc1 1 cсc $(\mathrm{S}(=\mathrm{O})(=\mathrm{O}) \mathrm{NN}=\mathrm{Cc} 2 \mathrm{c} 3 \mathrm{c}(\operatorname{ccc} 2 \mathrm{O}) \operatorname{cccc} 3) \mathrm{cc} 1$ & 55 \\
\hline 154 & $\mathrm{Clc} 1 \mathrm{ccc}(\mathrm{S}(=\mathrm{O})(=\mathrm{O}) \mathrm{NN}=\mathrm{Cc} 2 \mathrm{c} 3 \mathrm{c}(\operatorname{ccc} 2 \mathrm{OC}) \operatorname{cccc} 3) \mathrm{cc} 1$ & 19 \\
\hline 155 & $\operatorname{Clc} 1 \operatorname{ccc}(\mathrm{S}(=\mathrm{O})(=\mathrm{O}) \mathrm{NN}=\mathrm{Cc} 2 \mathrm{c} 3 \mathrm{c}(\operatorname{cc} 4 \mathrm{c} 2 \operatorname{cccc} 4) \operatorname{cccc} 3) \operatorname{cc} 1$ & 7 \\
\hline 156 & $\mathrm{~S}(=\mathrm{O})(=\mathrm{O})(\mathrm{NN}=\mathrm{Cc} 1 \mathrm{c} 2 \mathrm{c}(\operatorname{ccc} 1 \mathrm{O}) \operatorname{cccc} 2) \operatorname{c} 1 \mathrm{ccc}(\mathrm{F}) \mathrm{cc} 1$ & 150 \\
\hline 157 & $\mathrm{~S}(=\mathrm{O})(=\mathrm{O})(\mathrm{NN}=\mathrm{Cc} 1 \mathrm{c} 2 \mathrm{c}(\operatorname{ccc} 1 \mathrm{OC}) \operatorname{cccc} 2) \operatorname{c} 1 \mathrm{ccc}(\mathrm{F}) \mathrm{cc} 1$ & 55 \\
\hline 158 & $\mathrm{~S}(=\mathrm{O})(=\mathrm{O})(\mathrm{NN}=\mathrm{Cc} 1 \mathrm{c} 2 \mathrm{c}(\mathrm{cc} 3 \mathrm{c} 1 \mathrm{ccc} 3) \operatorname{cccc} 2) \mathrm{c} 1 \mathrm{ccc}(\mathrm{F}) \mathrm{cc} 1$ & 13.5 \\
\hline 159 & $\operatorname{sCC}(\mathrm{NC}(=\mathrm{O}) \mathrm{CCCC}(\mathrm{N}) \mathrm{C}(\mathrm{O})=\mathrm{O}) \mathrm{C}(=\mathrm{O}) \mathrm{NC}(\mathrm{C}(\mathrm{C}) \mathrm{C}) \mathrm{C}(\mathrm{O})=\mathrm{O}$ & 20 \\
\hline 160 & $\mathrm{SCC}(\mathrm{O})=\mathrm{O}$ & 1.3 \\
\hline 161 & $\operatorname{SCC}(O C)=O$ & 12 \\
\hline 162 & $\mathrm{SCC}(=\mathrm{O}) \mathrm{NC}$ & 12 \\
\hline 163 & $\operatorname{SCCC}(O)=O$ & 170 \\
\hline 164 & $\operatorname{sCCCC}(O)=0$ & 128 \\
\hline 165 & $\mathrm{SC}(\mathrm{C}(\mathrm{O})=\mathrm{O}) \mathrm{C}$ & 4.5 \\
\hline 166 & $\mathrm{SC}(\mathrm{C}(=\mathrm{O}) \mathrm{NCC}(\mathrm{O})=\mathrm{O}) \mathrm{C}$ & 15 \\
\hline 167 & SCCO & 200 \\
\hline 168 & SCCN & 120 \\
\hline 169 & SCCS & 6 \\
\hline 170 & $\mathrm{SC}(\mathrm{C}(\mathrm{S}) \mathrm{C}(\mathrm{O})=\mathrm{O}) \mathrm{C}(\mathrm{O})=\mathrm{O}$ & 20 \\
\hline 171 & $\mathrm{SC}(\mathrm{CO}) \mathrm{CS}$ & 13 \\
\hline 172 & $\mathrm{~S}(\mathrm{O})(=\mathrm{O})(=\mathrm{O}) \mathrm{CC}(\mathrm{S}) \mathrm{CS}$ & 11 \\
\hline 173 & Sc1 $\operatorname{ccccc} 1 \mathrm{C}(\mathrm{O})=\mathrm{O}$ & 200 \\
\hline 174 & Sc1ccccc1N & 200 \\
\hline 175 & $\operatorname{Sc} 1 \mathrm{cc}(\mathrm{C}(\mathrm{O})=\mathrm{O}) \mathrm{c}([\mathrm{N}+](=\mathrm{O})[\mathrm{O}-]) \mathrm{cc} 1$ & 500 \\
\hline 176 & SCc1сcсcс1 1 & 5 \\
\hline 177 & SCc1ccccc1CS & 2.3 \\
\hline 178 & $\mathrm{SCC}(\mathrm{O}) \mathrm{C}(\mathrm{O}) \mathrm{CS}$ & 10 \\
\hline 179 & $\mathrm{SCC}(\mathrm{N}) \mathrm{C}(\mathrm{O})=\mathrm{O}$ & 1050 \\
\hline 180 & $\operatorname{SCC}(\mathrm{N}) \mathrm{CC}(\mathrm{C}) \mathrm{C}$ & 75 \\
\hline 181 & Clc1cc2ncce(NCCNC(=O)CCS)c2cc1 & 14.2 \\
\hline 182 & Clc1cc2nccc $($ NCCCNC $(=0)$ CCS $)$ с2 2 cc1 & 6.60 \\
\hline 183 & Clc1cc2nccc(NCCCCNC $(=\mathrm{O}) \mathrm{CCS}) \mathrm{c} 2 \mathrm{cc} 1$ & 2.5 \\
\hline 184 & Clc1cc2nccc(NCCCCCNC $(=0) C C S) \operatorname{cocc1}$ & 6.70 \\
\hline 185 & Clc1cc2nccc(NCCCCCCNC(=O)CCS)c2cc1 & 3.40 \\
\hline 186 & $\operatorname{sCC}(\mathrm{Cc} 1 \operatorname{ccccc} 1) \mathrm{C}(\mathrm{O})=\mathrm{O}$ & 16.4 \\
\hline 187 & $\operatorname{SCC}(\operatorname{CCc} 1 \operatorname{ccccc} 1) \mathrm{C}(\mathrm{O})=\mathrm{O}$ & 7.40 \\
\hline 188 & $\operatorname{sCC}(\mathrm{CCC} 1 \operatorname{ccccc} 1) \mathrm{C}(\mathrm{O})=\mathrm{O}$ & 9.70 \\
\hline 189 & SCC $($ CCCC 1 1ссссс 1$) \mathrm{C}(\mathrm{O})=\mathrm{O}$ & 1.2 \\
\hline 190 & $\operatorname{SCCC}(\mathrm{Oc} 1 \mathrm{c}(\mathrm{F}) \mathrm{c}(\mathrm{F}) \mathrm{c}(\mathrm{F}) \mathrm{c}(\mathrm{F}) \mathrm{c} 1 \mathrm{~F})=\mathrm{O}$ & \\
\hline 191 & $\mathrm{~S}(\mathrm{C}(=\mathrm{O}) \mathrm{CCS}) \mathrm{CCC}(\mathrm{Oc} 1 \mathrm{c}(\mathrm{F}) \mathrm{c}(\mathrm{F}) \mathrm{c}(\mathrm{F}) \mathrm{c}(\mathrm{F}) \mathrm{c} 1 \mathrm{~F})=\mathrm{O}$ & \\
\hline 192 & $\mathrm{Oc} 1 \mathrm{cc}(\operatorname{cc}(\mathrm{c} 1) \mathrm{C}(\mathrm{O})=\mathrm{O}) \mathrm{C}(=\mathrm{O}) \mathrm{NO}$ & 67(\%IC50) \\
\hline 193 & Oc1cc $(\operatorname{cc}(\mathrm{c} 1) \mathrm{C}(=\mathrm{O}) \mathrm{NO}) \mathrm{C}(=\mathrm{O}) \mathrm{c} 1 \mathrm{ccc}(\mathrm{O}) \mathrm{cc} 1$ & 83(\%IC50) \\
\hline 194 & Oc1cc $(\mathrm{cc}(\mathrm{c} 1) \mathrm{C}(\mathrm{O})=\mathrm{O}) \mathrm{C}(=\mathrm{O}) \mathrm{c} 1 \mathrm{ccc}(\mathrm{O}) \mathrm{cc} 1$ & 100(\%IC50) \\
\hline 195 & Oc1cc $(\operatorname{cc}(\mathrm{c} 1) \mathrm{C}(=\mathrm{O}) \mathrm{NO}) \mathrm{C}(=\mathrm{O}) \mathrm{Nc} 1 \mathrm{ccccc} 1$ & $92(\%$ IC50) \\
\hline 196 & Oc1cc(cc(c1)C $(=\mathrm{O}) \mathrm{NO}) \mathrm{C}(=\mathrm{O}) \mathrm{NCc} 1 \mathrm{ccccc} 1$ & 81(\%IC50) \\
\hline 197 & Oc1cc $(\operatorname{cc}(\mathrm{c} 1) \mathrm{C}(=\mathrm{O}) \mathrm{NO}) \mathrm{C}(=\mathrm{O}) \mathrm{Nc} 1 \mathrm{cc}[\mathrm{nH}] \mathrm{c} 1$ & 85(\%IC50) \\
\hline 198 & Oc1cc $(\operatorname{cc}(\mathrm{c} 1) \mathrm{C}(=\mathrm{O}) \mathrm{NO}) \mathrm{C}(=\mathrm{O}) \mathrm{N}(\mathrm{CC}) \mathrm{CC}$ & 85(\%IC50) \\
\hline 199 & Oc1cc $(\operatorname{cc}(\mathrm{c} 1) \mathrm{C}(=\mathrm{O}) \mathrm{NO}) \mathrm{C}(=\mathrm{O}) \mathrm{NC}$ & $93(\%$ IC50) \\
\hline 200 & $\mathrm{~S}(=\mathrm{O})(=\mathrm{O})(\mathrm{NCCNC}(=\mathrm{O}) \mathrm{CCS}) \mathrm{c} 1 \mathrm{c} 2 \mathrm{c}(\operatorname{ccc} 1) \mathrm{c}(\mathrm{N}(\mathrm{C}) \mathrm{C}) \mathrm{ccc} 2$ & 5.20 \\
\hline 201 & $\mathrm{~S}(=\mathrm{O})(=\mathrm{O})(\mathrm{NCCCNC}(=\mathrm{O}) \mathrm{CCS}) \mathrm{c} 1 \mathrm{c} 2 \mathrm{c}(\mathrm{ccc} 1) \mathrm{c}(\mathrm{N}(\mathrm{C}) \mathrm{C}) \mathrm{ccc} 2$ & 6.30 \\
\hline
\end{tabular}




\begin{tabular}{|c|c|c|}
\hline 202 & $\mathrm{~S}(=\mathrm{O})(=\mathrm{O})(\mathrm{NCCCCNC}(=\mathrm{O}) \mathrm{CCS}) \mathrm{c} 1 \mathrm{c} 2 \mathrm{c}(\operatorname{ccc} 1) \mathrm{c}(\mathrm{N}(\mathrm{C}) \mathrm{C}) \mathrm{ccc} 2$ & 0.70 \\
\hline 203 & $\mathrm{~S}(=\mathrm{O})(=\mathrm{O})(\mathrm{NCCCCCNC}(=\mathrm{O}) \mathrm{CCS}) \mathrm{c} 1 \mathrm{c} 2 \mathrm{c}(\operatorname{ccc} 1) \mathrm{c}(\mathrm{N}(\mathrm{C}) \mathrm{C}) \operatorname{ccc} 2$ & 1.6 \\
\hline 204 & $\mathrm{~S}(=\mathrm{O})(=\mathrm{O})(\mathrm{NCCCCCCNC}(=\mathrm{O}) \mathrm{CCS}) \mathrm{c} 1 \mathrm{c} 2 \mathrm{c}(\mathrm{ccc} 1) \mathrm{c}(\mathrm{N}(\mathrm{C}) \mathrm{C}) \mathrm{ccc} 2$ & 1 \\
\hline 205 & $\mathrm{OC}(=\mathrm{O}) \operatorname{c} 1 \mathrm{cccnc} 1 \mathrm{C}(\mathrm{O})=\mathrm{O}$ & $\begin{array}{l}67 \\
\text { (Inhibition rate) }\end{array}$ \\
\hline 206 & $\mathrm{OC}(=\mathrm{O}) \mathrm{c} 1 \mathrm{cc}(\mathrm{ncc} 1) \mathrm{C}(\mathrm{O})=\mathrm{O}$ & $\begin{array}{l}78 \\
\text { (Inhibition rate) }\end{array}$ \\
\hline 207 & $\mathrm{OC}(=\mathrm{O}) \mathrm{c} 1 \mathrm{ccc}(\mathrm{nc} 1) \mathrm{C}(\mathrm{O})=\mathrm{O}$ & $\begin{array}{l}81 \\
\text { (Inhibition rate) }\end{array}$ \\
\hline 208 & $\mathrm{OC}(=\mathrm{O}) \mathrm{c} 1 \mathrm{nc}(\mathrm{ccc} 1) \mathrm{C}(\mathrm{O})=\mathrm{O}$ & $\begin{array}{l}72 \\
\text { (Inhibition rate) }\end{array}$ \\
\hline 209 & OC $(=0)$ c1ncccc1 & $\begin{array}{l}54 \\
\text { (Inhibition rate) }\end{array}$ \\
\hline 210 & $\mathrm{OC}(=\mathrm{O}) \mathrm{c} 1 \mathrm{ccncc} 1 \mathrm{C}(\mathrm{O})=\mathrm{O}$ & $\begin{array}{l}88 \\
\text { (Inhibition rate) }\end{array}$ \\
\hline 211 & $\mathrm{SCc} 1 \mathrm{cc}(\mathrm{cc}(\mathrm{c} 1) \mathrm{CS}) \mathrm{C}(\mathrm{O})=\mathrm{O}$ & $0.360(\mathrm{Ki})$ \\
\hline 212 & $\operatorname{SCC}(=0) \operatorname{c} 1$ cсcсc1 & $0.67(\mathrm{Ki})$ \\
\hline 213 & $\operatorname{SCCC}(=\mathrm{O}) \mathrm{NC}(\mathrm{C}(\mathrm{O})=\mathrm{O}) \mathrm{C}$ & $68(\mathrm{Ki})$ \\
\hline 214 & $\operatorname{SCCC}(=O) N C(C(O)=O) C$ & $7.5(\mathrm{Ki})$ \\
\hline 215 & $\operatorname{SCCC}(=0) \operatorname{NC}(\mathrm{C}(\mathrm{O})=\mathrm{O}) \mathrm{c} 1 \operatorname{ccccc} 1$ & $0.019(\mathrm{Ki})$ \\
\hline 216 & $\operatorname{SCCC}(=0) \operatorname{NC}(\mathrm{C}(\mathrm{O})=\mathrm{O}) \mathrm{c} 1 \operatorname{ccccc} 1$ & $0.019(\mathrm{Ki})$ \\
\hline 217 & $\operatorname{SCCC}(=0) \mathrm{NC}(\operatorname{Cc} 1 \operatorname{ccccc} 1) \mathrm{C}(\mathrm{O})=\mathrm{O}$ & $0.088(\mathrm{Ki})$ \\
\hline 218 & $\operatorname{SCCC}(=\mathrm{O}) \mathrm{NC}(\mathrm{C}(\mathrm{C}) \mathrm{C}) \mathrm{C}(\mathrm{O})=\mathrm{O}$ & $0.063(\mathrm{Ki})$ \\
\hline 219 & $\mathrm{OC}(=\mathrm{O}) \mathrm{C}(=\mathrm{C}(\mathrm{CC}) \mathrm{C}(\mathrm{O})=\mathrm{O}) \mathrm{CC}$ & 2.5 \\
\hline 220 & $\mathrm{OC}(=\mathrm{O}) \mathrm{C}(\mathrm{Cc} 1 \mathrm{ccccc} 1)=\mathrm{C}(\mathrm{C} C) \mathrm{C}(\mathrm{O})=\mathrm{O}$ & 0.29 \\
\hline 221 & $\mathrm{OC}(=\mathrm{O}) \mathrm{C}(\mathrm{C}(\mathrm{C}) \mathrm{C})=\mathrm{C}(\mathrm{C}(\mathrm{O})=\mathrm{O}) \mathrm{C}$ & 2.4 \\
\hline 222 & $\mathrm{OC}(=\mathrm{O}) \mathrm{C}(=\mathrm{C}(\mathrm{C}(\mathrm{C}) \mathrm{C}) \mathrm{C}(\mathrm{O})=\mathrm{O}) \mathrm{C} 1 \mathrm{CCCCC} 1$ & 0.19 \\
\hline 223 & $\mathrm{O} 1 \mathrm{CCC}(\mathrm{CC} 1) \mathrm{C}(=\mathrm{C}(\mathrm{C}(\mathrm{C}) \mathrm{C}) \mathrm{C}(\mathrm{O})=\mathrm{O}) \mathrm{C}(\mathrm{O})=\mathrm{O}$ & 1.9 \\
\hline 224 & $\mathrm{OC}(=\mathrm{O}) \mathrm{C}(\mathrm{Cc} 1 \mathrm{ccc} n \mathrm{1})=\mathrm{C}(\mathrm{C}(\mathrm{C}) \mathrm{C}) \mathrm{C}(\mathrm{O})=\mathrm{O}$ & 2.8 \\
\hline 225 & $\mathrm{~S}(\mathrm{C}(\mathrm{C}) \mathrm{C}) \mathrm{C}(=\mathrm{C}(\mathrm{CC}) \mathrm{C}(\mathrm{O})=\mathrm{O}) \mathrm{C}(\mathrm{O})=\mathrm{O}$ & 1.2 \\
\hline 226 & $\mathrm{OC}(=\mathrm{O}) \mathrm{C}(\mathrm{C} \operatorname{cocc}(\operatorname{cc} 1) \mathrm{C}(=\mathrm{O}) \mathrm{N})=\mathrm{C}(\mathrm{C}(\mathrm{C}) \mathrm{C}) \mathrm{C}(\mathrm{O})=\mathrm{O}$ & 1.4 \\
\hline 227 & Oc1 1 ccс $(\operatorname{cc} 1) C C(=\mathrm{C}(\mathrm{C}(\mathrm{C}) \mathrm{C}) \mathrm{C}(\mathrm{O})=\mathrm{O}) \mathrm{C}(\mathrm{O})=\mathrm{O}$ & 0.56 \\
\hline 228 & $\mathrm{O}(\mathrm{CCN}) \mathrm{c} 1 \mathrm{ccc}(\operatorname{cc} 1) \mathrm{CC}(=\mathrm{C}(\mathrm{C}(\mathrm{C}) \mathrm{C}) \mathrm{C}(\mathrm{O})=\mathrm{O}) \mathrm{C}(\mathrm{O})=\mathrm{O}$ & 0.98 \\
\hline 229 & Ic1ccc(S(=O)(=O)NCc2[nH]nnc2COCCC\#C)cc1 & $>56$ \\
\hline 230 & Ic1ccc(S(=O)(=O)NCc2[nH]nnc2COC)cc1 & $>56$ \\
\hline 231 & Oc1c2c(C(=O)c3c(C2=O)c(NCCNCCO $)$ ccc3NCCNCCO $)$ (O) $)$ cc1 & $>56$ \\
\hline 232 & $\mathrm{OC}(=\mathrm{O}) \mathrm{c} 1 \mathrm{c}(\mathrm{C}) \mathrm{c}(\operatorname{ccc} 1 \mathrm{C}(\mathrm{O})=\mathrm{O}) \mathrm{CCCC}$ & 16 \\
\hline 233 & $\mathrm{OC}(=\mathrm{O}) \operatorname{c} 1 \mathrm{c}(\operatorname{cccc} 1 \mathrm{C}) \mathrm{C}(\mathrm{O})=\mathrm{O}$ & 160 \\
\hline 234 & $\mathrm{OC}(=\mathrm{O}) \operatorname{c} 1 \mathrm{c}(\operatorname{cccc} 1 \mathrm{C}(\mathrm{O})=\mathrm{O}) \mathrm{CCCO}$ & 13.2 \\
\hline 235 & $\mathrm{OC}(=\mathrm{O}) \operatorname{c1c}(\operatorname{cccc} 1 \mathrm{C}(\mathrm{O})=\mathrm{O})-\mathrm{c} 1 \mathrm{ccccc} 1$ & 0.968 \\
\hline 236 & {$[\mathrm{Na}] \mathrm{Oc} 1 \operatorname{ccccc} 1-\operatorname{c} 1 \operatorname{cccc}(\mathrm{C}(\mathrm{O})=\mathrm{O}) \mathrm{c} 1 \mathrm{C}(\mathrm{O})=\mathrm{O}$} & 2.44 \\
\hline 237 & Oc1cc $(\operatorname{ccc} 1)-\operatorname{c1} \operatorname{cccc}(\mathrm{C}(\mathrm{O})=\mathrm{O}) \mathrm{c} 1 \mathrm{C}(\mathrm{O})=\mathrm{O}$ & 1.75 \\
\hline 238 & Oc1ccc $(\operatorname{cc} 1)-\operatorname{c1cccc}(\mathrm{C}(\mathrm{O})=\mathrm{O}) \mathrm{c} 1 \mathrm{C}(\mathrm{O})=\mathrm{O}$ & 1.55 \\
\hline 239 & $\mathrm{OC}(=\mathrm{O}) \operatorname{c} 1 \mathrm{c}(\operatorname{cccc} 1 \mathrm{C}(\mathrm{O})=\mathrm{O})-\mathrm{c} 1 \operatorname{ccccc} 1 \mathrm{C}(\mathrm{O})=\mathrm{O}$ & 207 \\
\hline 240 & $\mathrm{OC}(=\mathrm{O}) \operatorname{c} 1 \mathrm{c}(\operatorname{cccc} 1 \mathrm{C}(\mathrm{O})=\mathrm{O})-\operatorname{c} 1 \mathrm{cc}(\operatorname{ccc} 1) \mathrm{C}(\mathrm{O})=\mathrm{O}$ & 17.9 \\
\hline 241 & $\mathrm{OC}(=\mathrm{O}) \operatorname{c} 1 \mathrm{c}(\operatorname{cccc} 1 \mathrm{C}(\mathrm{O})=\mathrm{O})-\operatorname{c} 1 \operatorname{ccc}(\operatorname{cc} 1) \mathrm{C}(\mathrm{O})=\mathrm{O}$ & 225 \\
\hline 242 & $\mathrm{OC}(=\mathrm{O}) \operatorname{c} 1 \operatorname{cc}(\operatorname{ccc} 1 \mathrm{C}(\mathrm{O})=\mathrm{O}) \mathrm{CCCC}$ & 243 \\
\hline 243 & $\mathrm{OC}(=\mathrm{O}) \operatorname{c} 1 \operatorname{cc}(\operatorname{ccc} 1 \mathrm{C}(\mathrm{O})=\mathrm{O}) \mathrm{C}(\mathrm{C})(\mathrm{C}) \mathrm{C}$ & 300 \\
\hline 244 & $\mathrm{OC}(=\mathrm{O}) \operatorname{c} 1 \operatorname{cc}(\operatorname{ccc} 1 \mathrm{C}(\mathrm{O})=\mathrm{O})-\mathrm{c} 1 \operatorname{ccccc} 1$ & 300 \\
\hline 245 & $\mathrm{OC}(=\mathrm{O}) \operatorname{c} 1 \operatorname{ccccc} 1 \mathrm{C}(\mathrm{O})=\mathrm{O}$ & 100 \\
\hline 246 & $\mathrm{OC}(=\mathrm{O}) \operatorname{c} 1 \operatorname{cc}(\operatorname{ccc} 1 \mathrm{C}(\mathrm{O})=\mathrm{O}) \mathrm{C}$ & 300 \\
\hline 247 & Oc1cс2c $(\operatorname{cc} 1 \mathrm{C}(=\mathrm{O}) \mathrm{NN}=\mathrm{Cc} 1 \mathrm{c} 3 \mathrm{c}(\operatorname{cc} 4 \mathrm{c} 1 \mathrm{ccc} 4) \operatorname{ccc} 3) \operatorname{cccc} 2$ & 1.02 \\
\hline 248 & $\mathrm{O}(\operatorname{c} 1 \mathrm{cc}(\operatorname{ccc} 1) \mathrm{C}=\mathrm{NNC}(=\mathrm{O}) \mathrm{c} 1 \mathrm{cc} 2 \mathrm{c}(\operatorname{cc} 1 \mathrm{O}) \operatorname{cccc} 2) \mathrm{c} 1 \mathrm{ccccc} 1$ & 7 \\
\hline 249 & $\mathrm{O}(\mathrm{c} 1 \mathrm{cc}(\mathrm{ccc} 1) \mathrm{C}=\mathrm{NNC}(=\mathrm{O}) \mathrm{c} 1 \mathrm{ccc}(\mathrm{cc} 1) \mathrm{C}(\mathrm{C})(\mathrm{C}) \mathrm{C}) \mathrm{c} 1 \mathrm{ccccc} 1$ & 6.7 \\
\hline 250 & $\mathrm{O}(\mathrm{Cc} 1 \mathrm{cccc} 1) \operatorname{c} 1 \mathrm{ccc}(\operatorname{cc} 1 \mathrm{OC}) \mathrm{C}=\mathrm{NNC}(=\mathrm{O}) \operatorname{c1ccc}(\operatorname{cc} 1) \mathrm{C}(\mathrm{C})(\mathrm{C}) \mathrm{C}$ & 1.8 \\
\hline 251 & $\mathrm{O}(\mathrm{Cc} 1 \mathrm{cccc} 1) \operatorname{c} 1 \mathrm{ccccc} 1 \mathrm{C}=\mathrm{NNC}(=\mathrm{O}) \operatorname{c} 1 \operatorname{ccc}(\operatorname{cc} 1) \mathrm{C}(\mathrm{C})(\mathrm{C}) \mathrm{C}$ & 4.7 \\
\hline 252 & $\mathrm{O} 1 \mathrm{c} 2 \mathrm{cc}(\operatorname{ccc} 2 \mathrm{OC} 1) \mathrm{C}=\mathrm{NNC}(=\mathrm{O}) \operatorname{c1ccc}(\operatorname{cc} 1) \mathrm{C}(\mathrm{C})(\mathrm{C}) \mathrm{C}$ & 10 \\
\hline 253 & $\mathrm{O}=\mathrm{C}(\mathrm{NN}=\mathrm{Cc} 1 \operatorname{ccccc} 1[\mathrm{~N}+](=\mathrm{O})[\mathrm{O}-]) \operatorname{c} 1 \operatorname{ccc}(\operatorname{cc} 1) \mathrm{C}(\mathrm{C})(\mathrm{C}) \mathrm{C}$ & 10 \\
\hline 254 & Oc1 1 cсссс1C=NNC $(=0) \operatorname{coc} 1 \mathrm{ccnc} 1$ & 100 \\
\hline 255 & Oc1cccc $1 \mathrm{C}=\mathrm{NNC}(=\mathrm{O}) \mathrm{c} 1 \mathrm{ccncc} 1$ & 100 \\
\hline 256 & Oc1 $1 \operatorname{cccc} 1 \mathrm{C}(=\mathrm{O}) \mathrm{NN}=\mathrm{C} \operatorname{c} 1 \operatorname{ccccc} 1 \mathrm{O}$ & 40 \\
\hline 257 & $\mathrm{ClC} 1(\mathrm{Cl}) \mathrm{C} 2 \mathrm{SCC}(\mathrm{C} 2 \mathrm{C} 1=\mathrm{O}) \mathrm{C}(\mathrm{O})=\mathrm{O}$ & 1000 \\
\hline 258 & $\mathrm{~S} 1 \mathrm{CC}(\mathrm{C} 2 \mathrm{C} 1 \mathrm{CC} 2=\mathrm{O}) \mathrm{C}(\mathrm{O})=\mathrm{O}$ & 235 \\
\hline 259 & $\mathrm{ClC} 1(\mathrm{Cl}) \mathrm{C} 2 \mathrm{SC}=\mathrm{C}(\mathrm{C} 2 \mathrm{C} 1=\mathrm{O}) \mathrm{C}(\mathrm{O})=\mathrm{O}$ & 213 \\
\hline 260 & $\mathrm{~S} 1 \mathrm{C}=\mathrm{C}(\mathrm{C} 2 \mathrm{C} 1 \mathrm{CC} 2=\mathrm{O}) \mathrm{C}(\mathrm{O})=\mathrm{O}$ & 500 \\
\hline 261 & $\mathrm{ClC} 1(\mathrm{Cl}) \mathrm{C} 2 \mathrm{SC}(\mathrm{OC}) \mathrm{C}(\mathrm{C} 2 \mathrm{C} 1=\mathrm{O}) \mathrm{C}(\mathrm{O})=\mathrm{O}$ & 122 \\
\hline 262 & $\mathrm{ClC} 1(\mathrm{Cl}) \mathrm{C} 2 \mathrm{SC}(\mathrm{OC}) \mathrm{C}(\mathrm{C} 2 \mathrm{C} 1=\mathrm{O}) \mathrm{C}(\mathrm{O})=\mathrm{O}$ & 122 \\
\hline
\end{tabular}

Table S2. Molecular Descriptors used in this work

Descriptor class

Number of descriptors in Descriptors each class 
Subdivided Surface Areas

Atom Counts and Bond Counts

Kier\&Hall Connectivity and Kappa Shape Indices

Adjacency and Distance Matrix Descriptors

Pharmacophore Feature Descriptors

Partial Charge Descriptors

Potential Energy

Descriptors

Surface Area, Volume and Shape Descriptors

Conformation Dependent Charge Descriptors
SlogP_VSA0, SlogP_VSA1, SlogP_VSA2, SlogP_VSA3, SlogP_VSA4, SlogP_VSA5, SlogP_VSA6, SlogP_VSA7, SlogP_VSA8, SlogP_VSA9, SMR_VSA0, SMR_VSA1, SMR_VSA2, SMR_VSA3, SMR_VSA4, SMR_VSA5, SMR_VSA6, SMR_VSA7

a_aro, a_count, a_heavy, a_ICM, a_IC, a_nH, a_nC, a_nN, a_nO, a_nF, a_nS, a_nI a_nCl, b_1rotN, b_1rotR, b_ar, b_count, b_double, b_heavy, b_rotR, b_rotN, b_single, chiral, chiral_u, lip_acc, lip_don, lip_drglike, lip_violation, opr_brigid, opr_leadlike, opr_nring, opr_nrot, opr_violation, rings, VAdjMa, VAdjEq

chi0, chi0_C, chi1, chi1_C, chi0v, chi0v_C, chi1v, chi1v_C, Kier1, Kier2, Kier3, KierA1, KierA2, KierA3, KierFlex, zagreb

balabanJ, BCUT_PEOE_0, BCUT_PEOE_1, BCUT_PEOE_2, BCUT_PEOE_3, BCUT_SLOGP_0, BCUT_SLOGP_1, BCUT_SLOGP_2, BCUT_SLOGP_3, BCUT_SMR_0, BCUT_SMR_1, BCUT_SMR_2, BCUT_SMR_3, diameter, petitjean, GCUT_PEOE_0, GCUT_PEOE_1, GCUT_PEOE_2, GCUT_PEOE_3, GCUT_SLOGP_0, GCUT_SLOGP_1, GCUT_SLOGP_2, GCUT_SLOGP_3, GCUT_SMR_0, GCUT_SMR_1, GCUT_SMR_2, GCUT_SMR_3, petitjeanSC, radius, VDistEq, VDistMa, wienerPath, wienerPol

a_acc, a_base, a_don, a_hyd, vsa_acc, vsa_don, vsa_hyd, vsa_other, vsa_pol, vsa_base

PEOE_PC+, PEOE_PC-, PEOE_RPC+, PEOE_RPC-, Q VSA_POS, PEOE_VSA_POS, PEOE_VSA_NEG, PEOE_VSA_PPOS, PEOE_VSA_PNEG, Q_VSA_HYD, PEOE_VSA_HYD, PEOE_VSA_POL, PEOE_VSA_FPOS, PEOE_VSA_FNEG, PEOE_VSA_FPPOS, PEOE_VSA_FPNEG, PEOE_VSA_FHYD, PEOE_VSA_FPOL, PEOE_VSA+6, PEOE_VSA+5, PEOE_VSA+4, PEOE_VSA+3, PEOE_VSA+2, PEOE_VSA+1, PEOE_VSA+0, PEOE_VSA-0, PEOE_VSA-1, PEOE_VSA-2, PEOE_VSA-3, PEOE_VSA-4, PEOE_VSA-5, PEOE_VSA-6

E, E_ang, E_ele, E_nb, E_sol, E_stb, E_str, E_strain, E_tor, E_vdw

ASA, dens, pmi, pmiX, pmiY, pmi1, pmi2, pmi3, npr1, npr2, rgyr, std_dim1, std_dim2, vol, VSA, vsurf_V, vsurf_S, vsurf_R, vsurf_G, vsurf_W*(8 descriptors), vsurf_IW*(8 descriptors), vsurf_CW*(8 descriptors), vsurf_EWmin*(3 descriptors), vsurf_DW*(3 descriptors), vsurf_D*(8 descriptors), vsurf_ID*(8 descriptors), vsurf_EDmin*(3 descriptors), vsurf_DD*(3 descriptors), vsurf_HL*(2 descriptors), vsurf_A, vsurf_CP, vsurf_Wp*(3 descriptors), vsurf_HB*(8 descriptors) 